\title{
An Implicit Stabilized Finite Element Method For The Compressible Navier-Stokes Equations Using Finite Calculus
}

\author{
Mohammad Kouhi*1 and Eugenio Oñate ${ }^{1,2}$ \\ ${ }^{1}$ Centre Internacional de Mètodes Numèrics en Enginyeria (CIMNE), Barcelona, Spain \\ ${ }^{2}$ Universitat Politècnica de Catalunya (UPC), Barcelona, Spain \\ * Corresponding author: e-mail: kouhi@cimne.upc.edu
}

November 25, 2014

\begin{abstract}
A new implicit stabilized formulation for the numerical solution of the compressible NavierStokes equations is presented. The method is based on the Finite Calculus (FIC) scheme using the Galerkin finite element method (FEM) on triangular grids. Via the FIC formulation, two stabilization terms, called streamline term and transverse term, are added to the original conservation equations in the space-time domain. The non-linear system of equations resulting from the spatial discretization is solved implicitly using a damped Newton method benefiting from the exact Jacobian matrix. The matrix system is solved at each iteration with a preconditioned GMRES method. The efficiency of the proposed stabilization technique is checked out in the solution of $2 \mathrm{D}$ inviscid and laminar viscous flow problems where appropriate solutions are obtained especially near the boundary layer and shock waves. The work presented here can be considered as a follow up of a previous work of the authors [24]. In that paper, the stabilized Galerkin FEM based on the FIC formulation was derived for the Euler equations together with an explicit scheme. In the present paper, the extension of this work to the Navier-Stokes equations using an implicit scheme is presented.
\end{abstract}

Keywords: High-Speed Compressible Flows; Navier-Stokes Equations; Stabilized Finite Element; Finite Calculus, Implicit Scheme

\section{Introduction}

Stabilization of numerical methods is an important research topic for high-speed compressible flows modeled by the Navier-Stokes equations. Basically, stabilization strategies intend to avoid the occurrence of numerical instabilities which normally have two main sources, the high value of convective terms in the original partial differential equation and the sharp gradients and shocks in localized zones of the solution. Also, these techniques should be able to predict the boundary layer, properly.

Based on the FEM, several stabilized formulations for compressive flows have been developed during the last decades. Donea [13] originally derived a class of stabilized FEM using the LaxWendroff/Taylor-Galerkin scheme. Based on the idea of the artificial diffusion, Hughes et al. $[17,18]$ extended the classical Streamline-Upwind/Petrov-Galerkin (SUPG), initially proposed by Brooks and Hughes [4] for incompressible flows. The SUPG method has been widely used by several authors for compressible flows $[1,6,7,40,47]$. Other popular stabilization techniques for compressible flows were based on the Galerkin least squares (GLS) method $[8,15,46]$ which coincides with the original SUPG method under some specified conditions. Using of the fractional step concept $[9,10]$, Zienkiewicz and co-workers introduced the characteristic-based split (CBS) method [11, 50-52], which benefited from the anisotropic shock capturing term presented by Codina [12]. Another artificial diffusion schemes were developed by Peraire et al. [38], Morgan 
et al. [29] and Zienkiewicz and $\mathrm{Wu}$ [49]. Based on the idea of limiters, Boris and Book [3] developed the Flux Corrected Transport (FCT) and Löhner et al. [26] extended this scheme to unstructured meshes in the FEM. Recently, the so-called variational multiscale (VMS) method, originally introduced by Hughes [19] has been successfully applied to derive stabilized finite element formulations for flow problems [14, 20, 40-44]. Based on the finite volume (FV) scheme and following the idea of artificial diffusion, an important numerical improvement was conducted by Jameson et al. [22] using a series of second and fourth order stabilization methods. The study of finite volume flux vector splitting was presented by Anderson et al. [2] where several advantages of the MUSCL-type approach over standard flux-differencing scheme were discussed. Unified stabilized FEM formulations for compressible and incompressible flows were presented by Hauke and Hughes [15] and Mittal and Tezduyar [28].

Within the family of stabilization techniques, the Finite Increment Calculus (FIC, or Finite Calculus in short) formulation has been successfully implemented for the stabilization of advectivediffusive transport and incompressible fluid flow problems by Oñate and co-workers [30-37]. In this paper, a FIC-based stabilized formulation for the numerical solution of the compressible Navier-Stokes equations is considered in the context of Galerkin FEM using an implicit scheme. In a previous work [24], we proposed an explicit FIC-FEM formulation for the numerical solution of the Euler equations.

The FIC technique is based on writing the balance of fluxes in the momentum, mass balance and energy conservation equations in a space-time domain of finite size. It aims to preventing the creation of instabilities that usually appear in the numerical solution of fluid flow problems due to the high convective terms and sharp gradients. This leads to a modified non-local form of the standard governing equations in mechanics that incorporate additional residual terms that depend on characteristic lengths in space and time. In the context of the compressible flow equations, the FIC approach introduces two stabilization terms, called the streamline term and the transverse term. Generally, the streamline term takes care of the instabilities produced from the convective terms while the transverse term smooths the solution in the high gradient zones. An implicit algorithm is implemented to overcome the stability limitations depending on the mesh size. This implicit algorithm takes advantage of Newton method for solving of the non-linear system of equations using the exact Jacobian matrix. The final linear system of equations is solved at each iteration with a preconditioned GMRES method.

In this work we explore the advantages of the FIC formulation to provide appropriate numerical solutions for the compressible Navier-Stokes equations in conjunction with an implicit solver. Some numerical test examples related to inviscid and laminar viscous flows are presented. By studying the quality of solutions near shock waves, the boundary layer and the stagnation point it is found that the usual oscillations observed in the Galerkin FEM [52], especially near high gradient zones, are eliminated by implementing the FIC stabilization terms without introducing an excessive numerical dissipation.

The layout of the paper is the following: In Section 2 the compressible Navier-Stokes equations are described. Section 3 presents the derivation of the stabilized formulation based on the FIC scheme. The spatial discretization of the proposed stabilized formulation via the FEM and the solution method for steady state problems are presented in Section 4. Numerical results for inviscid and laminar viscous flows in subsonic, transonic and supersonic regimes are shown in Section 5. Finally, conclusions and general remarks are summarized in Section 6.

\section{Governing Equations}

The two-dimensional (2D) compressible Navier-Stokes equations, obtained from the combination of the mass balance, momentum and energy equations, can be written in the following conservative form:

$$
\frac{\partial \mathbf{U}}{\partial t}+\frac{\partial \mathbf{F}_{i}}{\partial x_{i}}-\frac{\partial \mathbf{G}_{i}}{\partial x_{i}}=\mathbf{0} \text { for } i=1,2
$$


where $\mathbf{U}, \mathbf{F}$ and $\mathbf{G}$ are the vectors of conservative variables, inviscid fluxes and viscous fluxes, respectively which can be expressed as

$$
\mathbf{U}=\left[\begin{array}{c}
\rho \\
\rho v_{1} \\
\rho v_{2} \\
\rho e
\end{array}\right] \quad \mathbf{F}_{i}=\left[\begin{array}{c}
\rho v_{i} \\
\rho v_{1} v_{i}+p \delta_{i 1} \\
\rho v_{2} v_{i}+p \delta_{i 2} \\
v_{i}(p+\rho e)
\end{array}\right] \quad \mathbf{G}_{i}=\left[\begin{array}{c}
0 \\
\sigma_{1 i} \\
\sigma_{2 i} \\
k \frac{\partial T}{\partial x_{i}}+v_{j} \sigma_{i j}
\end{array}\right]
$$

where $\rho, \mathbf{v}, p, T$, and $e$ are the density, the velocity vector, the static pressure, the absolute temperature, and the total internal energy per unit mass, respectively. $\sigma$ is the viscous stress tensor, $k$ is the thermal conductivity coefficient and $\delta_{i j}$ is the Kronecker delta. In the above equations $i, j=1, n_{d}$ with $n_{d}$ is the number of space dimensions ( $n_{d}=2$ for $2 \mathrm{D}$ flow problems).

The Navier-Poisson law for a Newtonian fluid expresses the components of the viscous stress tensor $\boldsymbol{\sigma}$ in term of the velocity. For an isotropic media

$$
\sigma_{i j}=\mu\left(\frac{\partial v_{i}}{\partial x_{j}}+\frac{\partial v_{j}}{\partial x_{i}}\right)+\lambda \frac{\partial v_{k}}{\partial x_{k}} \delta_{i j} \text { for } k=1,2
$$

where a bulk viscosity of $(3 \lambda+2 \mu)=0$ is assumed. In Equation $(3) \mu=\mu(T)$ is the dynamic viscosity coefficient which is calculated from Sutherland's equation [16].

The standard sum convention for terms with repeated indices is adopted in the paper, unless otherwise specified.

By defining $\gamma=1.4$ as the ratio of specific heats, the assumed equation of state for an ideal gas has the following form

$$
p=(\gamma-1) \rho\left(e-0.5 v_{j} v_{j}\right)
$$

The Euler equations for a non-viscous fluid can be recovered from the Navier-Stokes equations (Equations (1)) by neglecting the viscous stress and the heat conduction terms. i.e. $\mathbf{G}_{i}=\mathbf{0}$.

\section{Derivation of the Stabilized Formulation}

In this section, we derive the FIC-based stabilized formulation for the mass, momentum and energy equations is presented. Although the same idea is applied to construct the stabilized formulation for each component, the strategy used for the momentum and energy equations is somehow different from the one implemented for the mass equation. This difference is due to the implementation of the FIC scheme in space for stabilization of the momentum and energy equations, while a space-time FIC scheme is used to stabilize the mass equation.

\subsection{FIC Scheme for Navier-Stokes Equations}

The FIC-based stabilized formulation for the Navier-Stokes equations is obtained by writing the mass balance equation in a space-time domain of finite size using higher order Taylor series expansions as $[30,33,35]$

\section{Mass Balance}

$$
r_{d}-\frac{1}{2} \mathbf{h}_{d} \cdot \nabla r_{d}+\frac{1}{2} \tau_{d} \frac{\partial r_{d}}{\partial t}=0
$$

The same idea is implemented for the momentum and energy balance equations in a space domain of finite size as

\section{Momentum}

$$
r_{m_{i}}-\frac{1}{2} \mathbf{h}_{m_{i}} \cdot \nabla r_{m_{i}}=0
$$

Energy

$$
r_{e}-\frac{1}{2} \mathbf{h}_{e} \cdot \nabla r_{e}=0
$$


In Equations (5), (6) and (7) $\mathbf{h}_{d}, \mathbf{h}_{m_{i}}$ and $\mathbf{h}_{e}$ are characteristic length vectors, $\tau_{d}$ is a stabilization parameter and $r_{d}, r_{m_{i}}$ and $r_{e}$ are the residuals of the mass equation, the $i$ th momentum equation and the energy equation, respectively, defined as

$$
\begin{gathered}
r_{d}:=\frac{\partial \rho}{\partial t}+\frac{\partial\left(\rho v_{j}\right)}{\partial x_{j}}=0 \\
r_{m_{i}}:=\frac{\partial\left(\rho v_{i}\right)}{\partial t}+\frac{\partial}{\partial x_{j}}\left(\rho v_{i} v_{j}\right)+\frac{\partial p}{\partial x_{i}}-\frac{\partial}{\partial x_{j}}\left(\sigma_{i j}\right)=0 \\
r_{e}:=\frac{\partial(\rho e)}{\partial t}+\frac{\partial}{\partial x_{j}}\left(v_{j}(\rho e+p)\right)-\frac{\partial}{\partial x_{j}}\left(\sigma_{i j} v_{i}+k \frac{\partial T}{\partial x_{j}}\right)=0
\end{gathered}
$$

with $i, j=1, n_{d}$.

More details on the definition of Equations (5), (6) and (7) can be found in [30]. Other applications of the FIC scheme to incompressible flows and convection-diffusion problems are presented in [31-35].

The time derivative term $\frac{\partial r_{d}}{\partial t}$ in Equation (5) needs to be modified in order to provide the required stability. For this reason, $r_{d}$ from Equation (8) is substituted in the time-derivative term of Equation (5). By neglecting the term $\frac{1}{2} \tau_{d} \frac{\partial^{2} \rho}{\partial t^{2}}$ from the deduced equation, the stabilized final mass balance equation has the form

$$
r_{d}-\frac{1}{2} \mathbf{h}_{d} \cdot \nabla r_{d}+\frac{1}{2} \tau_{d} \frac{\partial}{\partial x_{i}} \frac{\partial\left(\rho v_{i}\right)}{\partial t}=0
$$

The term $\frac{\partial\left(\rho v_{i}\right)}{\partial t}$ in the above equation can be expressed in term of the terms of Equation (9) as

$$
-\frac{\partial\left(\rho v_{i}\right)}{\partial t}=\frac{\partial\left(\rho v_{i} v_{j}\right)}{\partial x_{j}}+\frac{\partial p}{\partial x_{i}}-\frac{\partial}{\partial x_{j}}\left(\sigma_{i j}\right)=\nabla .\left(\mathbf{F}_{m_{i}}-\mathbf{G}_{m_{i}}\right)
$$

where $\boldsymbol{\nabla} .\left(\mathbf{F}_{m_{i}}-\mathbf{G}_{m_{i}}\right)$ is the divergence of the flux terms corresponding to the $i$ th momentum equation.

Substituting Equation (12) into (11) the FIC-based form of the mass equation can be expressed as

$$
r_{d}-\frac{1}{2} \mathbf{h}_{d} \cdot \nabla r_{d}-\frac{1}{2} \tau_{d} \boldsymbol{\nabla} \cdot\left(\boldsymbol{\nabla} \cdot\left(\mathbf{F}_{m}-\mathbf{G}_{m}\right)\right)=0
$$

It is to be mentioned that although the neglected term $\frac{1}{2} \tau_{d} \frac{\partial^{2} \rho}{\partial t^{2}}$ in Equation (11) can be obtained using an explicit finite difference scheme, we have found that this term has not an important role for steady state flow problems. However, we suggest to consider this term for unsteady cases.

\subsection{Definition of the Stabilization Parameters}

As shown in Equations (13), (6) and (7), the modified governing equations via the FIC method introduce naturally an additional term into the standard mass, momentum and energy equations through some stabilization parameters, namely characteristic length vectors $\mathbf{h}_{d}, \mathbf{h}_{m_{i}}$ and $\mathbf{h}_{e}$, as well as the pseudo-time stabilization parameter $\tau_{d}$.

Stabilization of the Navier-Stokes equations can be achieved by an appropriate definition of these parameters in such a way that unstable solutions, usually created in the numerical simulations of high-speed flow problems due to the high convective terms and sharp gradients, disappear. For this purpose, the characteristic length vectors $\mathbf{h}_{m_{i}}$ and $\mathbf{h}_{e}$ corresponding to the momentum and energy equations are defined as

$$
\begin{gathered}
\mathbf{h}_{m_{i}}=\beta_{m_{i}} \ell \frac{\mathbf{v}}{|\mathbf{v}|+v_{c}}+\left(1-\beta_{m_{i}}\right) \ell \frac{\boldsymbol{\nabla} v_{i}}{\left|\boldsymbol{\nabla} v_{i}\right|} \operatorname{sgn}\left(r_{m_{i}}\right) \\
\mathbf{h}_{e}=\beta_{e} \ell \frac{\mathbf{v}}{|\mathbf{v}|+v_{c}}+\left(1-\beta_{e}\right) \ell \frac{\boldsymbol{\nabla} T}{|\nabla T|} \operatorname{sgn}\left(r_{e}\right)
\end{gathered}
$$


where $\beta_{m_{i}}$ and $\beta_{e}$ are constant parameters ranging between zero and one, $\ell$ is a characteristic element size corresponding to the momentum and energy equations, $\operatorname{sgn}($.$) denotes the sign function,$ $|\mathbf{v}|$ is the modulus of the velocity vector and $v_{c}=\sqrt{\gamma \frac{p}{\rho}}$ is the speed of the sound in the flow.

In Equations (14) and (15) the characteristic length vector corresponding to each equation is defined as the summation of two terms. The first one is the streamline stabilization term. This term handles the instabilities due to the high convective terms by adding extra diffusion in the direction of the velocity. On the other hand, the instabilities generated near zones with some sharp gradients are treated via the second term, called transverse stabilization term. This term introduces an isotropic (residual-based) diffusion matrix.

The same idea is applied for deriving of the characteristic length vector $\mathbf{h}_{d}$ and the pseudo-time stabilization parameter $\tau_{d}$ corresponding to the mass equation. Their expression is

$$
\mathbf{h}_{d}=\left(1-\beta_{d}\right) \ell \frac{\nabla \rho}{|\nabla \rho|} \operatorname{sgn}\left(r_{d}\right) \quad \tau_{d}=\beta_{d} \frac{\ell}{|\mathbf{v}|+v_{c}}
$$

where $0 \leq \beta_{d} \leq 1$. Comparing Equations (13) and (16), we see that parameters $\tau_{d}$ and $\mathbf{h}_{d}$ in the stabilized formulation of the mass equation have the same functionality as the streamline stabilization term and the transverse stabilization term, respectively, introduced for the momentum and energy equations.

\subsection{General Stabilized Formulation}

By substituting the stabilization parameters from Equations (16), (14) and (15) into Equations (13), (6) and (7), the general FIC-based stabilized formulation for the compressible Navier-Stokes equations is obtained as

\section{Mass balance}

$$
r_{d}-\frac{1}{2}\left(1-\beta_{d}\right) \ell \operatorname{sgn}\left(r_{d}\right) \frac{\boldsymbol{\nabla} \rho}{|\boldsymbol{\nabla} \rho|} \cdot \boldsymbol{\nabla} r_{d}-\frac{1}{2} \beta_{d} \ell \frac{1}{|\mathbf{v}|+v_{c}} \boldsymbol{\nabla} \cdot\left(\boldsymbol{\nabla} \cdot\left(\mathbf{F}_{m}-\mathbf{G}_{m}\right)\right)=0
$$

\section{Momentum}

$$
r_{m i}-\frac{1}{2}\left(1-\beta_{m_{i}}\right) \ell \operatorname{sgn}\left(r_{m i}\right) \frac{\boldsymbol{\nabla} v_{i}}{\left|\boldsymbol{\nabla} v_{i}\right|} \cdot \nabla r_{m_{i}}-\frac{1}{2} \beta_{m_{i}} \ell \frac{\mathbf{v}}{|\mathbf{v}|+v_{c}} \cdot \nabla r_{m_{i}}=0
$$

Energy

$$
r_{e}-\frac{1}{2}\left(1-\beta_{e}\right) \ell \operatorname{sgn}\left(r_{e}\right) \frac{\boldsymbol{\nabla} T}{|\nabla T|} \cdot \nabla r_{e}-\frac{1}{2} \beta_{e} \ell \frac{\mathbf{v}}{|\mathbf{v}|+v_{c}} \cdot \nabla r_{e}=0
$$

Note that expressing the stabilization terms as a function of the residuals of the corresponding balance equations (See Equations (17), (18) and (19)), the consistency of the proposed FIC method is enforced.

\subsection{Selection of the stabilization parameters}

The rationale behind the choice of the stabilization parameters $\beta_{m_{i}}$ and $\beta_{e}$ is that they should account for streamline and transverse diffusion effects. Note that for $\beta_{m_{i}}, \beta_{e}=0$ the original SUPG stabilization scheme for the momentum and energy balance equations is recovered, where a stabilization diffusion is added in the direction of the velocity. On the other hand, $\beta_{m_{i}}, \beta_{e}=1$ introduces an isotropic diffusion term only.

A possible choice for choosing these parameters is

$$
\beta_{m_{i}}=1-\frac{\mathbf{v} \cdot \nabla v_{i}}{|\mathbf{v}|\left|\nabla v_{i}\right|} \quad, \quad \beta_{e}=1-\frac{\mathbf{v} \cdot \boldsymbol{\nabla} T}{|\mathbf{v}||\nabla T|}
$$

Note that for situations when the velocity field is orthogonal to the gradient $\nabla v_{i}$ then $\beta_{m_{i}}=1$, whereas $\beta_{m_{i}}=0$ if $\mathbf{v}$ is parallel to $\boldsymbol{\nabla} v_{i}$. The same occurs for $\beta_{e}$ when $\mathbf{v}$ is orthogonal or parallel to $\nabla T$. 
The form of Eqs.(20) introduces a non-linearity in the computation of $\beta_{m_{i}}$ and $\beta_{e}$. This can be overcome in transient problems by assuming that $\beta_{m_{i}}$ and $\beta_{d}$ are constant within a time step and equal to the value computed for the previous time increment.

For the steady state problems considered in this work we have assumed that the stabilization parameters $\beta_{m_{i}}$ and $\beta_{e}$ are constant throughout the non linear solution (i.e. $\beta_{m_{i}}=\beta_{d}=\beta$. This assumption simplifies the convergence of the iterative process.

Accurate results have been obtained for all the problems solved with $\beta=0.5$. This choice was based on the good results obtained with this assumption for inviscid compressible flow problem in [24]. The effect of choosing different values for $\beta$ on the quality of the results is studied in [24] as well as in the example presented in Section 5.2.

As for the stabilization parameter $\beta_{d}$ in the mass balance equation, in this work the simplest choice $\beta_{d}=\beta$ has been made. The optimal definition of $\beta_{d}$ is an open research topic.

The FEM is implemented in this work for the discretization of equations in space. Hence the characteristic element size is defined as $\ell=\left(2 \Omega^{e}\right)^{1 / 2}$ where $\Omega^{e}$ is the element area for $2 \mathrm{D}$ problems. Clearly, for $\ell \rightarrow 0$ the standard infinitesimal form of the balance Equations (8), (9) and (10) is recovered from the general stabilized formulation.

\section{Numerical Solution}

We present the spatial discretization of the stabilized Navier-Stokes equations (17)-(19) as well as the methodology implemented for solving the resulted system of algebraic equations for the steady state case.

\subsection{Spatial Discretization}

The well known Galerkin FEM [52] is implemented to discretize the FIC-based stabilized formulation in space. Vector $\mathbf{U}$ containing the conservative variables is approximated by a combination of continuous linear shape functions as

$$
\mathbf{U} \simeq \overline{\mathbf{U}}=\sum_{J=1}^{n} \mathbf{N}_{J} \overline{\mathbf{U}}_{J}
$$

where vector $\overline{\mathbf{U}}$ contains the approximate values of the conservative variables. $\mathbf{N}$ is the matrix of interpolating shape functions, subscript index $J$ represents the values for the $J^{\text {th }}$ node and $n=3$ for linear triangles.

Let us assume a problem domain $\Omega$ with a boundary $\Gamma$. The standard weighted residual method is applied to Equations (17), (18) and (19), the stabilization terms are integrated by parts and the boundary terms are neglected. This yields the variational form of the discretized equations as

$$
\int_{\Omega} \mathbf{W} \overline{\mathbf{r}} d \Omega+\sum_{e}^{n_{e l}} \int_{\Omega_{e}} \frac{1}{2} \mathbf{S} \frac{\partial \mathbf{W}}{\partial x_{i}} \frac{\partial \overline{\mathbf{U}}}{\partial x_{i}} d \Omega+\sum_{e}^{n_{e l}} \int_{\Omega_{e}} \frac{\tau}{2} \mathbf{B}_{i} \frac{\partial \mathbf{W}}{\partial x_{i}} \overline{\mathbf{r}}_{s t} d \Omega=0
$$

where $n_{e l}$ is the number of elements, $i=1,2$ (for $2 \mathrm{D}$ problems) and $\mathbf{W}$ denotes the standard weighting function vector.

In Equation (22), the residual vectors $\overline{\mathbf{r}}$ and $\overline{\mathbf{r}}_{s t}$ as well as the vector of approximated primitive variables $\overline{\tilde{\mathbf{U}}}$ have the following form

$$
\overline{\mathbf{r}}=\left[\begin{array}{c}
\bar{r}_{d} \\
\bar{r}_{m_{1}} \\
\bar{r}_{m_{2}} \\
\bar{r}_{e}
\end{array}\right] \quad \overline{\mathbf{r}}_{s t}=\left[\begin{array}{c}
1 \\
\bar{r}_{m_{1}} \\
\bar{r}_{m_{2}} \\
\bar{r}_{e}
\end{array}\right] \quad \overline{\mathbf{U}}=\left[\begin{array}{c}
\bar{\rho} \\
\bar{v}_{1} \\
\bar{v}_{2} \\
\bar{T}
\end{array}\right]
$$

where $\bar{r}_{d}, \bar{r}_{m_{i}}$ and $\bar{r}_{e}$ denote the approximate finite element residuals for the mass, momentum and energy equations, respectively. 
In Equation (23), $\tau=\frac{\beta \ell}{|\bar{v}|+\bar{v}_{c}}$ is the the stabilization parameter and the stabilization matrices $\mathbf{S}$ and $\mathbf{B}_{i}$ in Eq.(22) have the following form (for $\beta_{m_{i}}=\beta_{e}=\beta_{d}=\beta$ )

$$
\mathbf{S}=(1-\beta) \ell\left[\begin{array}{cccc}
\frac{\left|\bar{r}_{d}\right|}{|\nabla \bar{\rho}|} & 0 & 0 & 0 \\
0 & \frac{\left|\bar{r}_{m_{1}}\right|}{\left|\nabla \bar{v}_{1}\right|} & 0 & 0 \\
0 & 0 & \frac{\left|\bar{r}_{m_{2}}\right|}{\left|\nabla \bar{v}_{2}\right|} & 0 \\
0 & 0 & 0 & \frac{\left|\bar{r}_{e}\right|}{|\nabla T|}
\end{array}\right] \quad \mathbf{B}_{i}=\left[\begin{array}{cccc}
\nabla \cdot\left(\overline{\mathbf{F}}_{m_{i}}-\overline{\mathbf{G}}_{m_{i}}\right) & 0 & 0 & 0 \\
0 & \bar{v}_{i} & 0 & 0 \\
0 & 0 & \bar{v}_{i} & 0 \\
0 & 0 & 0 & \bar{v}_{i}
\end{array}\right]
$$

where $\bar{v}_{i}$ is the $i$ th component of the nodal velocity vector and $\boldsymbol{\nabla} \cdot\left(\overline{\mathbf{F}}_{m}-\overline{\mathbf{G}}_{m}\right)$ is the divergence of the approximate finite element flux terms corresponding to the momentum equation.

The Galerkin form of the discretized equations is obtained by making the weighting functions equal to the interpolation ones $(\mathbf{W}=\mathbf{N})$. The final step of the discretization is to apply integration by parts to the first term of Equation (22) to yield the weak form as

$$
\begin{gathered}
\int_{\Omega} \mathbf{N} \frac{\partial \overline{\mathbf{U}}}{\partial t} d \Omega-\int_{\Omega} \frac{\partial \mathbf{N}}{\partial x_{i}}\left(\overline{\mathbf{F}}_{i}-\overline{\mathbf{G}}_{i}\right) d \Omega+\int_{\Gamma} \mathbf{N}\left(\overline{\mathbf{F}}_{n}-\overline{\mathbf{G}}_{n}\right) d \Gamma \\
-\sum_{e}^{n_{e l}} \int_{\Omega_{e}} \frac{1}{2} \mathbf{S} \frac{\partial \mathbf{N}}{\partial x_{i}} \frac{\partial \overline{\mathbf{U}}}{\partial x_{i}} d \Omega-\sum_{e}^{n_{e l}} \int_{\Omega_{e}} \frac{\tau}{2} \mathbf{B}_{i} \frac{\partial \mathbf{N}}{\partial x_{i}} \overline{\mathbf{r}}_{s t} d \Omega=0
\end{gathered}
$$

with $i=1,2$. The first integral in Equation (25) corresponds to the time derivative part of the Navier-Stokes equation, the second integral is the contribution of the inviscid and viscous flux terms to the weak form and the third integral represents the boundary flux terms. The elemental contributions of the streamline and transverse stabilization terms are delivered by the fourth and fifth integrals, respectively.

Terms $\overline{\mathbf{F}}_{i}$ and $\overline{\mathbf{G}}_{i}$ in Equation (25) represent the $i$ th components of the approximated vectors corresponding to the inviscid flux vector $\mathbf{F}$ and the viscous flux vector $\mathbf{G}$, respectively (See Equation (2)). Also, $\overline{\mathbf{F}}_{n}=\overline{\mathbf{F}}_{i} n_{i}$ and $\overline{\mathbf{G}}_{n}=\overline{\mathbf{G}}_{i} n_{i}$ are the projections of vectors $\overline{\mathbf{F}}$ and $\overline{\mathbf{G}}$, respectively, along the normal vector to the boundary $\mathbf{n}=\left[n_{1}, n_{2}\right]^{T}$ (for $2 \mathrm{D}$ problems). The different types of the boundary conditions are defined in Section 4.3.

The following remarks are made:

- By considering the sign of the residuals in the definition of the characteristic length vectors $\mathbf{h}_{d}, \mathbf{h}_{m_{i}}$ and $\mathbf{h}_{e}$ in Equations (16), (14) and (15), all the components of matrix $\mathbf{S}$ are positive, yielding a positive value of the shock capturing diffusion.

- For the linear interpolation implemented here, the derivative of the viscous flux term $\overline{\mathbf{G}}_{m_{i}}$ in $\boldsymbol{\nabla} \cdot\left(\overline{\mathbf{F}}_{m}-\overline{\mathbf{G}}_{m}\right)$ appearing in the definition of the stabilization matrix $\mathbf{B}_{i}$ (Equation (24)) is zero.

\subsection{Solution Method}

For the Navier-Stokes computations, an implicit scheme is needed due to the small size of the elements in the normal direction to the boundary layer.

For the steady-state problems solved in this work we will neglect the temporal derivative term (i.e. the first term) in Equation (25). Assembling the elemental contributions from Equation (25), the global system of non-linear equations can be written for the steady state case as

$$
\mathbf{R}(\overline{\mathbf{U}})=\mathbf{0}
$$

with

$$
\begin{gathered}
\mathbf{R}_{I}(\overline{\mathbf{U}})=-\int_{\Omega} \frac{\partial \mathbf{N}_{I}}{\partial x_{i}}\left(\overline{\mathbf{F}}_{i}-\overline{\mathbf{G}}_{i}\right) d \Omega+\int_{\Gamma} \mathbf{N}_{I}\left(\overline{\mathbf{F}}_{n}-\overline{\mathbf{G}}_{n}\right) d \Gamma \\
-\sum_{e}^{n_{e l}} \int_{\Omega_{e}} \frac{\tau}{2} \mathbf{B}_{i} \frac{\partial \mathbf{N}_{I}}{\partial x_{i}} \overline{\mathbf{r}}_{s t} d \Omega-\sum_{e}^{n_{e l}} \int_{\Omega_{e}} \frac{1}{2} \mathbf{S} \frac{\partial \mathbf{N}_{I}}{\partial x_{i}} \frac{\partial \overline{\tilde{\mathbf{U}}}}{\partial x_{i}} d \Omega
\end{gathered}
$$


where $\mathbf{R}_{I}(\overline{\mathbf{U}})$ denotes the non-linear steady-state residual vector corresponding to the $I$ th global node.

The final global system of non-linear equations is solved for $\overline{\mathbf{U}}$ using a standard Newton method as

$$
\begin{aligned}
& {\left[\frac{\partial \mathbf{R}}{\partial \overline{\mathbf{U}}}\right]^{n} \Delta \overline{\mathbf{U}}^{n}=-\mathbf{R}\left(\overline{\mathbf{U}}^{n}\right)} \\
& \Delta \overline{\mathbf{U}}^{n}=\overline{\mathbf{U}}^{n+1}-\overline{\mathbf{U}}^{n}
\end{aligned}
$$

with $n$ being the iteration number.

In the above equation $\left[\frac{\partial \mathbf{R}}{\partial \mathbf{U}}\right]$ is the Jacobian matrix calculated by differentiation of the residual vector $\mathbf{R}(\overline{\mathbf{U}})$ with respect to the numerical solution $\overline{\mathbf{U}}$. It involves the linearization of all the terms contributing to the residual vector $\mathbf{R}(\overline{\mathbf{U}})$ (See Equation (27)). In the current work the linearization of the inviscid and viscous flux terms is handled through the implementation an inviscid flux Jacobian matrix $\mathbf{A}_{i}$ and the diffusivity matrix $\mathbf{K}_{i j}$, presented in [23], whereas a hand-coded linearization is implemented for the streamline and transverse stabilization terms. Following this idea, the general form of the Jacobian matrix can be expressed as

$$
\begin{aligned}
{\left[\frac{\partial \mathbf{R}_{I}}{\partial \overline{\mathbf{U}}_{J}}\right]=} & -\int_{\Omega} \frac{\partial \mathbf{N}_{I}}{\partial x_{i}}\left(\mathbf{A}_{i} \mathbf{N}_{J}-\mathbf{K}_{i j} \frac{\partial \mathbf{N}_{J}}{\partial x_{j}}\right) d \Omega+\int_{\Gamma} \mathbf{N}_{I}\left(\mathbf{A}_{i} \mathbf{N}_{J}-\mathbf{K}_{i j} \frac{\partial \mathbf{N}_{J}}{\partial x_{j}}\right) n_{i} d \Gamma \\
& -\sum_{e}^{n_{e l}} \int_{\Omega_{e}} \frac{1}{2} \frac{\partial \mathbf{N}_{I}}{\partial x_{i}}\left[\frac{\partial \tau}{\partial \overline{\mathbf{U}}_{J}} \mathbf{B}_{i} \overline{\mathbf{r}}_{s t}+\tau \frac{\partial \mathbf{B}_{i}}{\partial \overline{\mathbf{U}}_{J}} \overline{\mathbf{r}}_{s t}+\tau \mathbf{B}_{i} \frac{\partial \overline{\mathbf{r}}_{s t}}{\partial \overline{\mathbf{U}}_{J}}\right] d \Omega \\
& -\sum_{e}^{n_{e l}} \int_{\Omega_{e}} \frac{1}{2} \frac{\partial \mathbf{N}_{I}}{\partial x_{i}}\left[\frac{\partial \mathbf{S}}{\partial \overline{\mathbf{U}}_{J}} \frac{\partial \overline{\tilde{\mathbf{U}}}}{\partial x_{i}}+\mathbf{S} \frac{\partial\left(\frac{\partial \overline{\mathbf{U}}}{\partial x_{i}}\right)}{\partial \overline{\mathbf{U}}_{J}}\right] d \Omega
\end{aligned}
$$

In order to preserve the divergence of Newton method due to the inappropriate initial guess and the creation of sharp gradients, it is common to augment the Jacobian matrix $\left[\frac{\partial \mathbf{R}}{\partial \tilde{\mathbf{U}}}\right]$ with a damping term based on the mass matrix $\mathbf{M}$ as

$$
\begin{aligned}
& {\left[\frac{\delta_{I J}}{\Delta t_{I}} \mathbf{M}_{i j}+\frac{\partial \mathbf{R}_{I}}{\partial \overline{\mathbf{U}}_{J}}\right]^{n} \Delta \overline{\mathbf{U}}_{J}^{n}=-\mathbf{R}_{I}\left(\overline{\mathbf{U}}^{n}\right)} \\
& \text { with } \mathbf{M}_{i j}=\int_{\Omega} \mathbf{N}_{I} \mathbf{N}_{J} d \Omega
\end{aligned}
$$

with $I, J=1, N$ where $N$ is the total number of nodes in the mesh. In the above equation, the nodal (pseudo) time step $\Delta t_{I}$ is the minimum of the time steps corresponding to the elements connected to node $I$. For inviscid problems, the time step for an element $e$ can be completed as

$$
\Delta t_{e}=C F L \frac{h}{|\overline{\mathbf{v}}|+\bar{v}_{c}}
$$

where $C F L$ denotes the allowable Courant-Friedrichs-Lewy number and $h=\ell$ is the characteristic element size. Note that $C F L$ is a global number, while the remaining variables in the above equation are calculated at the element level. Including the viscous terms, the time step for an element can be obtained as

$$
\Delta t_{e}=C F L \frac{h}{|\overline{\mathbf{v}}|+\bar{v}_{c}+\frac{4 \mu \gamma^{3 / 2} M_{\infty}}{\bar{\rho}_{m i n} \operatorname{Pr} R e_{\infty} h}}
$$

In the above equation, $\bar{\rho}_{\min }$ is the minimum density within the element, $M_{\infty}$ is the free stream Mach number, $\operatorname{Pr}$ is the non dimensional Prandtl number and $R e_{\infty}$ is the free stream Reynold 
number. The other variables have the same meaning as defined above. Details can be found in [16]. The Prandtl number is assumed to be constant and equal to 0.72 .

In this work the $C F L$ is responsible for adding a scalable damping term during the start-up computational process and has the following form

$$
C F L(n)=\min \left(\alpha^{n} C F L(0), C F L(\max )\right)
$$

where $C F L(0)=0.01, C F L(\max )=10^{12}$ and $\alpha$ is selected between 1.001 and 1.01 depending on the problem. Using this pattern for the $C F L$ value, and increasing the pseudo time step to a large value $\left(C F L=10^{12}\right)$ the damped unsteady terms vanish in Eq.(30) and the desired quadratic convergence rate of the Newton method leading to the final steady state solution is achieved.

The linear system of equations (30) consisting of a sparse block matrix is solved using the Generalized Minimum Residual (GMRES) method described in [39]. In order to improve the convergence, the preconditioned GMRES algorithm based on a block-diagonal preconditioning has been implemented in this work.

\subsection{Boundary Conditions}

\subsubsection{Euler Equations}

Equation (25) assumes a computational domain $\Omega$ surrounded by a boundary $\Gamma$ with unit normal n. So far, the algorithm only describes the contributions of each element across the integral $\Omega$ but does not yet states how to incorporate the boundary conditions.

In our work, two types of boundaries have been considered for Euler-type (inviscid) flows: the slip boundary $\Gamma_{W}$ through which mass flux is not possible, and the far field (inflow/outflow) boundary $\Gamma_{\infty}$ through which mass flux is possible. The boundary condition must be applied in a compatible form with the equations to be solved.

\section{Slip Boundary}

The normal component of the velocity must vanish on the boundary. This condition can be enforced in a weak form by setting the inviscid flux across the boundary in Equation (25) to:

$$
\mathbf{v} . \mathbf{n}=0 \Rightarrow \overline{\mathbf{F}}_{n}=\left[\begin{array}{c}
0 \\
p n_{1} \\
p n_{2} \\
0
\end{array}\right]
$$

where $n_{1}$ and $n_{2}$ are the component of the unit normal vector to the boundary $\mathbf{n}=\left[n_{1}, n_{2}\right]^{T}$.

\section{Far Field Boundary}

Depending on the flow regime, the components of the solution which enter the domain are to be enforced and the ones leaving the domain have to be set free. By using Roe approximation for Riemann solvers, the boundary flux for a node $I$ on the far field boundary is computed as

$$
\overline{\mathbf{F}}_{n}^{I}=\frac{1}{2}\left\{\overline{\mathbf{F}}_{n}\left(\overline{\mathbf{U}}^{I}\right)+\overline{\mathbf{F}}_{n}\left(\overline{\mathbf{U}}^{\infty}\right)-\left|\overline{\mathbf{A}}_{n}\left(\overline{\mathbf{U}}^{I}, \overline{\mathbf{U}}^{\infty}\right)\right|\left(\overline{\mathbf{U}}^{I}-\overline{\mathbf{U}}^{\infty}\right)\right\}
$$

where superscript $\infty$ represents the freestream value and $\overline{\mathbf{A}}_{n}\left(\overline{\mathbf{U}}^{I}, \overline{\mathbf{U}}^{\infty}\right)$ is the Roe matrix computed in the direction normal to the boundary. More details about the derivation of the Roe matrix can be found in $[16,48]$.

\subsubsection{Navier-Stokes Equations}

In general, the treatment of the boundary condition for the Navier-Stokes equations is similar to the one for the Euler equations. However, the steady momentum and energy equations are elliptic and their modeling is more complex. Details are given in [16]. 


\section{No-Slip Boundary}

For the Navier-Stokes equations, in addition to the conditions on the velocity, some conditions must be considered for the temperature. The physical no-slip boundary conditions for the velocity is

$$
v_{i}=0
$$

where $i=1,2$. This condition can be enforced by assigning appropriate Dirichlet boundary conditions for the momentum components of $\overline{\mathbf{U}}$. As for the temperature boundary conditions, if an adiabatic wall is modeled then the heat flux $q_{n}$ across the wall is zero, i.e.

$$
q_{n}=-k \frac{\partial T}{\partial n}=0
$$

which can be set weakly on the viscous boundary flux $\overline{\mathbf{G}}_{n}$. For an isothermal wall, this condition yields the Dirichlet boundary conditions for the energy components of $\overline{\mathbf{U}}$ as

$$
T=T_{W} \Rightarrow \rho e-\rho c_{v} T_{W}=0
$$

where $T_{W}$ is a specified wall temperature. Note that since $\rho$ is not given a priori, the condition (38) should be updated during the solution process.

\section{Far Field Boundary}

For a node $I$ located at the far field boundary, the flux $\overline{\mathbf{G}}_{n}^{I}$ for a node $I$ belonging to the far field boundary can be obtained numerically by applying the far field values at the boundary, i.e. $\overline{\mathbf{G}}_{n}^{I}=\overline{\mathbf{G}}_{n}^{\infty}$.

\section{$5 \quad$ Test Examples}

In this section, a set of numerical examples is presented in order to evaluate the performance of the FIC-based stabilization method. In the first example, corresponding to an inviscid subsonic flow, the capability of the proposed stabilized formulation in conjunction with the implicit scheme is assessed. The rest of examples study different viscous flow regimes. For each example the computations start using the upstream values as the initial solution and they are advanced until a fully convergence of the residual vector $\mathbf{R}(\overline{\mathbf{U}})$ to machine zero (1E-14 for double precision) is obtained. The numerical results are compared with published results.

A value of $\beta=0.5$ has been used in all the examples. The sensitivity of the numerical solution to $\beta$ is studied in Example 5.2.

\subsection{Example I: Subsonic Inviscid Flow Past a Bump}

A popular example for subsonic regime is the bump problem consisting in an inviscid flow with Mach 0.35 pasting a bump with the maximum thickness of 0.08 in a rectangle domain of height 2 and length 4. In order to demonstrate the effect of grid resolution on the behavior of the proposed scheme, the domain is discretized uniformly by setting three different values for the element size. The generated unstructured meshes, called coarse, intermediate and fine mesh, have 1902, 3712 and 7454 elements, respectively. The coarse mesh is shown in Figure 1. The slip boundary condition is applied on the upper and lower sides of the domain, whereas the far field boundary condition is considered on the left and right sides. 


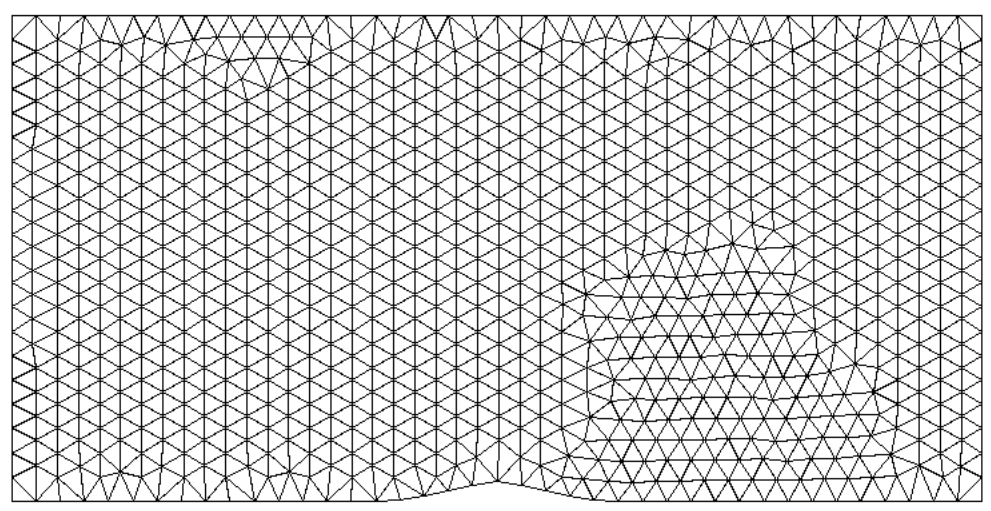

Figure 1: Subsonic inviscid flow past a bump example. The generated unstructured coarse mesh.

Figures 2 and 3 display the density and pressure coefficient contours, respectively, corresponding to the different discretizations which indicate the smoothness of the solution in all the domain. It can be seen that although the FIC method is capable to predicting appropriate results by using a coarse discretization, the smoothness of the numerical solutions is enhanced by improving the quality of the mesh. 


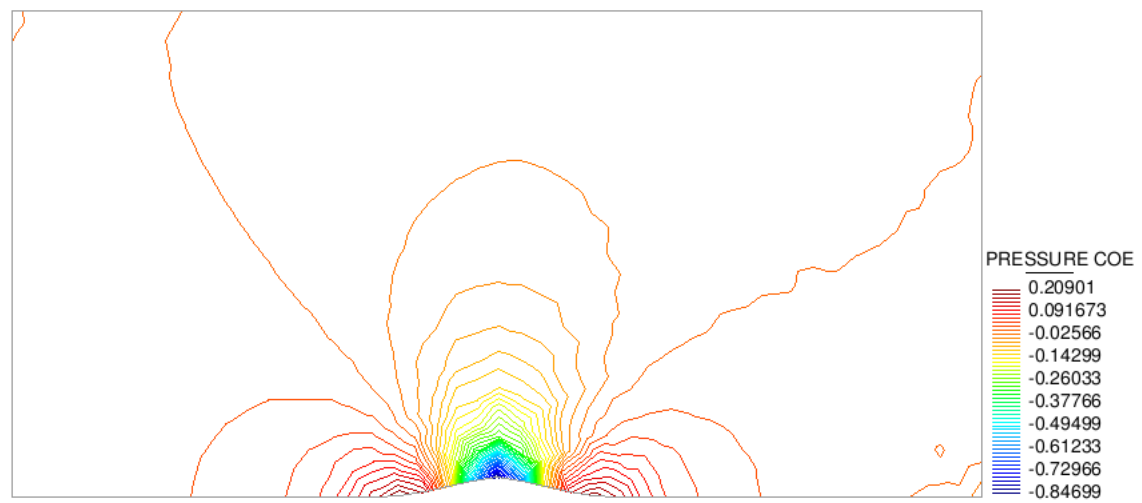

(a)

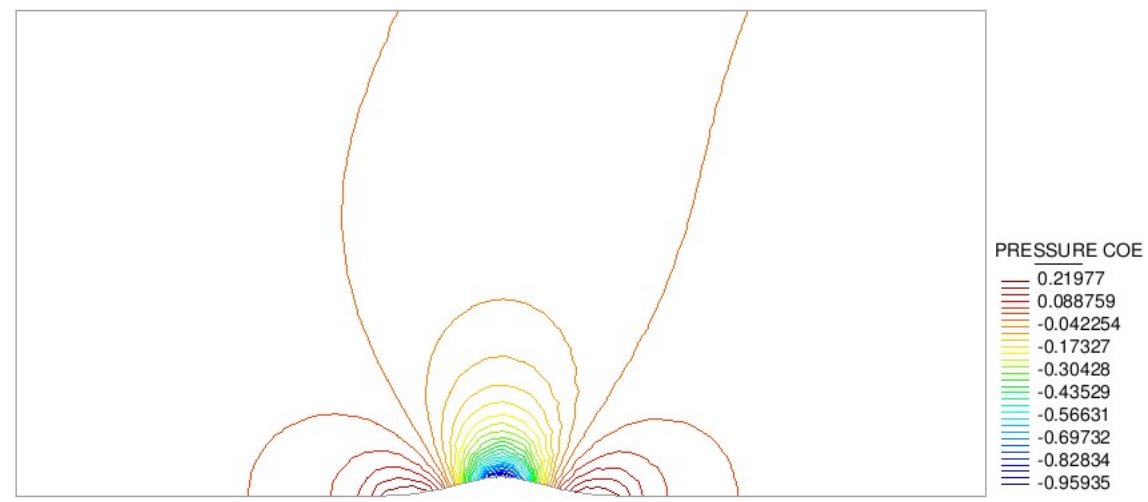

(b)

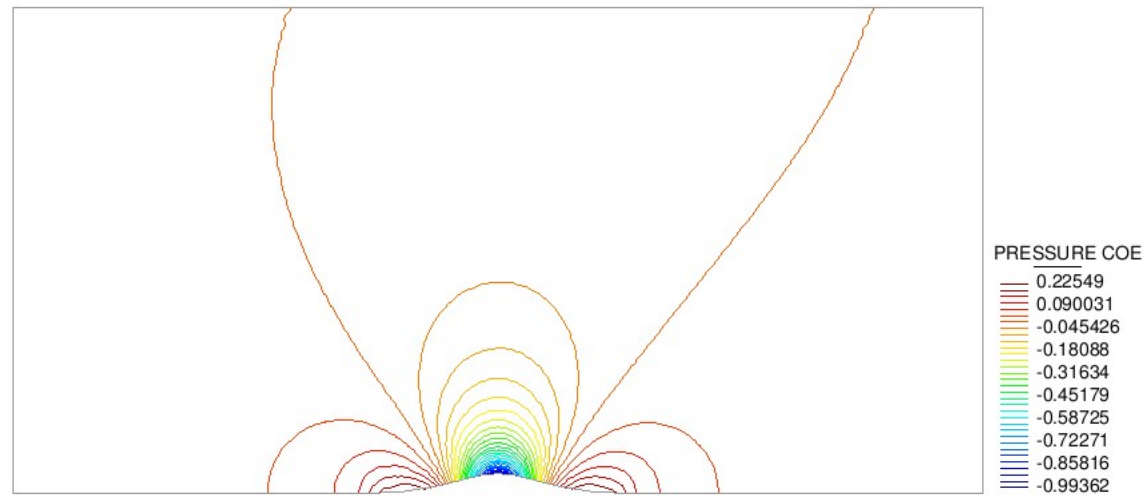

(c)

Figure 3: Subsonic inviscid flow past a bump example. Pressure coefficient contours for (a) coarse mesh, (b) intermediate mesh and (c) fine mesh.

The iterative convergence histories for the different meshes using Newton method are plotted in Figure 4. Computations are continued until a suitable convergence for the residual to machine zero precision is obtained. It can be observed that as initial transient flow passes, the damping term vanishes and quadratic convergence is achieved for all discretizations. 


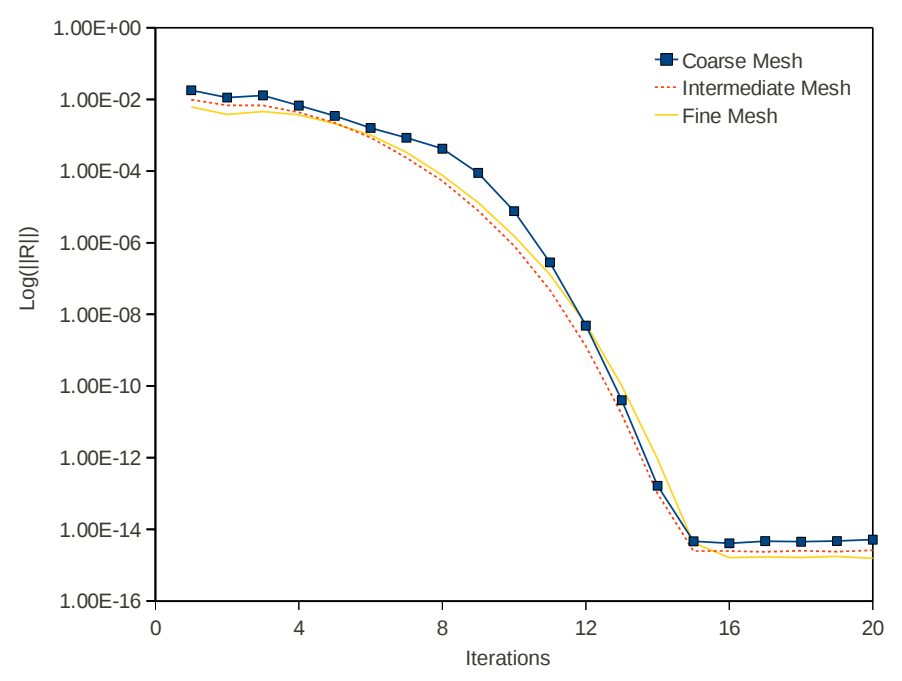

Figure 4: Subsonic inviscid flow past a bump example. Convergence history of Newton method using different discretizations.

\subsection{Example II: Subsonic Viscous Flow Past a NACA0012 Airfoil}

The subsonic viscous flow around a NACA0012 airfoil is presented here for demonstrating the behavior of the developed stabilized formulation in the viscous regime. The flow conditions are $R e=5000, M_{\infty}=0.5$ and $\alpha=0^{\circ}$ and a circular computational domain with the radius of 8 chords is considered. The assumed circular domain is discretized into 12623 nodes and 25300 3noded triangles including a structured mesh of 15 layers near the airfoil boundary which is merged with an unstructured mesh in the remaining of the computational domain. For the first layer of elements at the boundary, the normal element size has the value of 0.0005 which is increased by a geometric progression for the following layers. The details of the mesh near the airfoil are shown in Figure 5. The no slip adiabatic wall condition is imposed at the airfoil surface, whereas the far field condition is applied at the outer boundary.

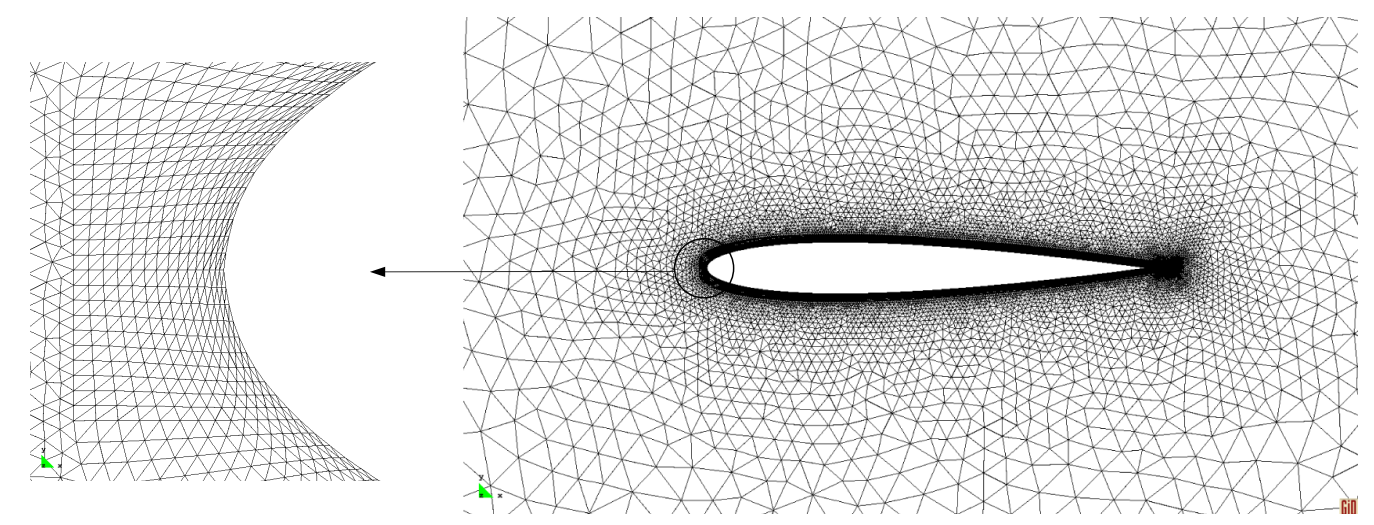

Figure 5: Subsonic viscous flow past NACA0012 airfoil. Detail of the mesh in the vicinity of the airfoil.

Results for the Mach number contours are presented in Figure 6 showing an overall excellent agreement with the reference values [27]. Figure 7a illustrates the recirculation bubble at the trailing edge. Each vector of the figure represents the modulus and direction of velocity at each node of the mesh. Pressure contours are shown in Figure $7 \mathrm{~b}$. The fact that the lines are parallel to each other with almost no oscillations indicates the good quality of the results. 


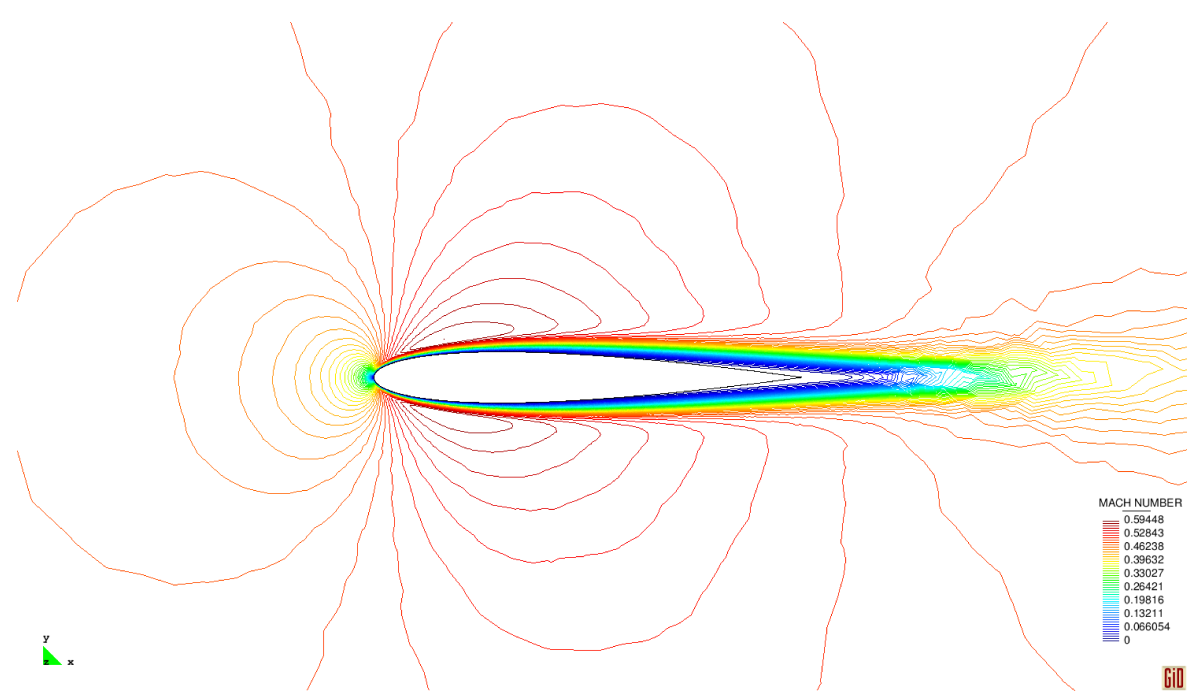

Figure 6: Subsonic viscous flow past a NACA0012 airfoil. Mach number contours.

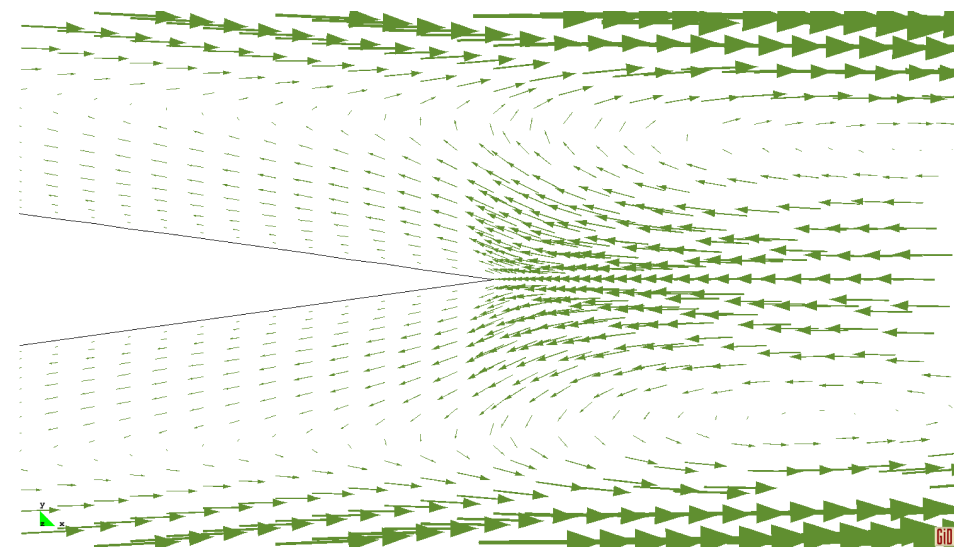

(a)

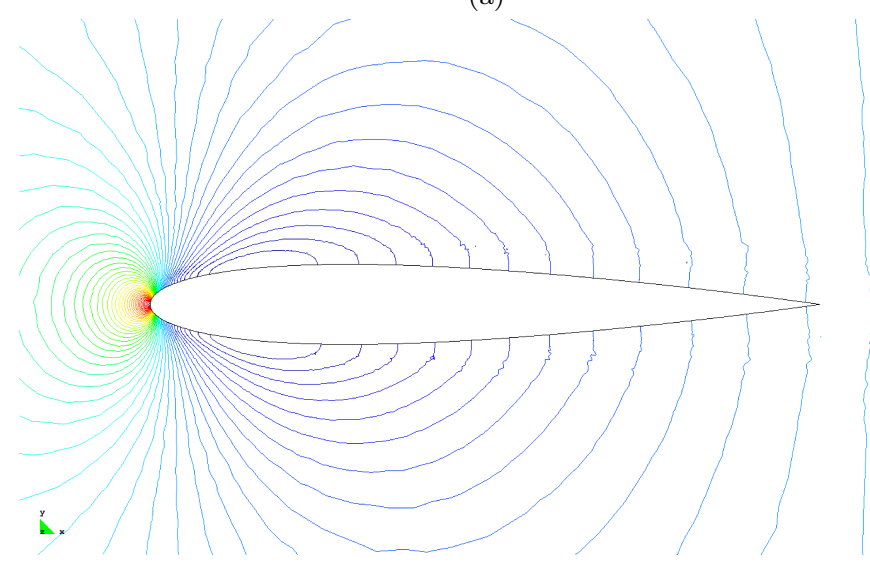

(b)

Figure 7: Subsonic viscous flow past a NACA0012 airfoil. (a) Close-up of computed velocity vectors near the trailing edge and (b) Details of pressure contours.

A more severe test of accuracy is the plot of the pressure coefficient $c_{p}$ and the skin friction coefficient $c_{f}$ along the airfoil, presented in Figures 8 and 9, respectively, showing the agreement of the obtained results with the reference values [27]. It is to be noted that the peak value of $c_{f}$ 
is slightly underestimated. Better results can be obtained by using a finer mesh near the leading edge.

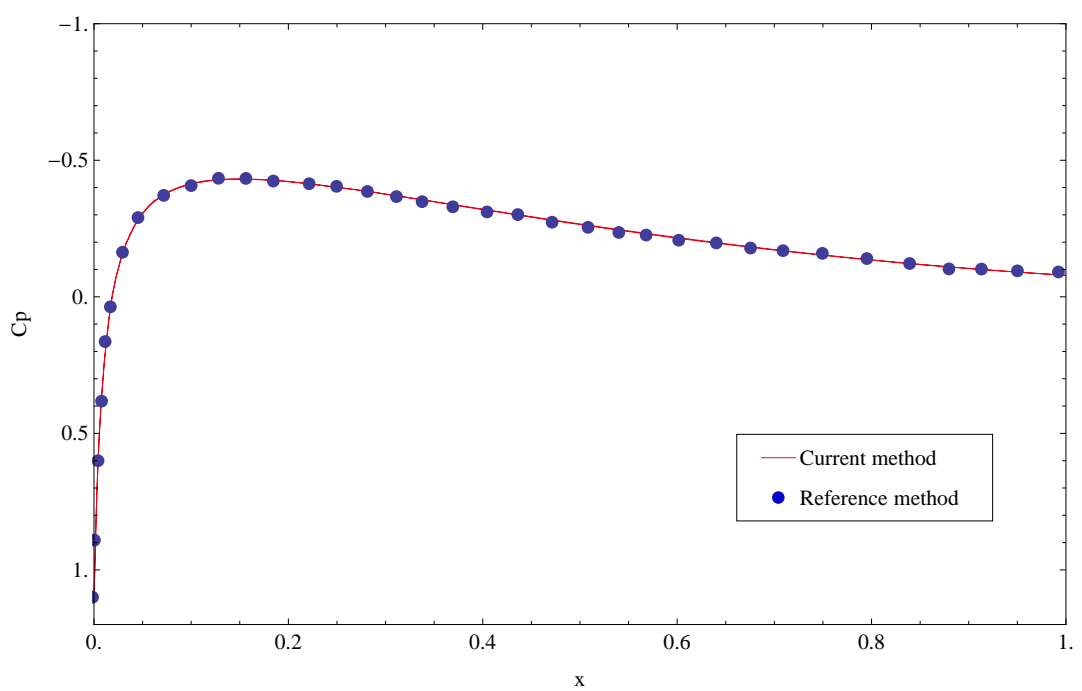

Figure 8: Subsonic viscous flow past a NACA0012 airfoil. Comparison of the obtained pressure coefficient $C_{p}$ distribution with the numerical results of reference [27].

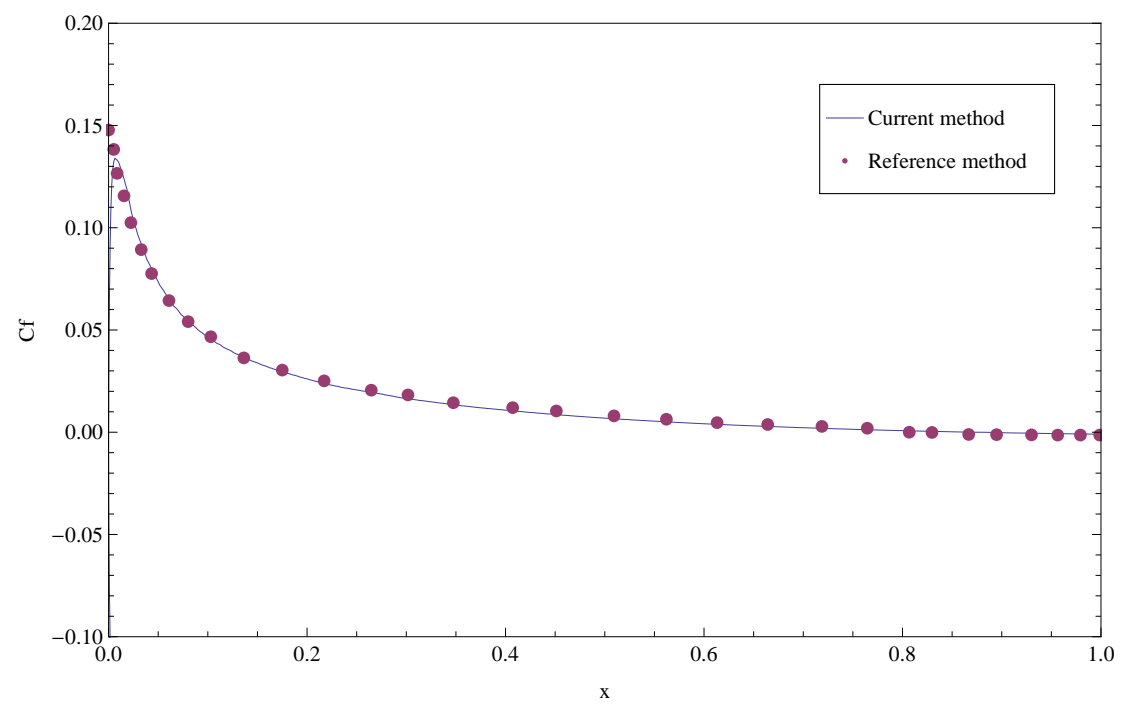

Figure 9: Subsonic viscous flow past a NACA0012 airfoil. Comparison of the obtained skin-friction coefficient $C_{f}$ distribution with the numerical results of reference [27].

The variation of the accuracy and the convergence with the change in the coefficient $\beta$ is investigated in this example. Table 1 presents an estimate of the solution accuracy as measured by the computed values of the location of the separation point using three different values of 0.25 , 0.50 and 0.75 for $\beta$. It can be found that the values obtained with $\beta=0.25$ and $\beta=0.50$ have a good agreement with the results presented in the reference paper [27], ranging from $80.9 \%-83.4 \%$ chord. 
Table 1: Subsonic viscous flow past a NACA0012 airfoil. Comparison of separation location values obtained from different values of $\beta$.

\begin{tabular}{|c|ccc|}
\hline & $\beta=0.25$ & $\beta=0.50$ & $\beta=0.75$ \\
\hline Separation Location & $82.4 \%$ & $83.0 \%$ & $91.0 \%$ \\
\hline
\end{tabular}

The variation of the convergence history of the density at the stagnation point with the change in $\beta$ is presented in Figure 10 showing that the choice of $\beta=0.5$ does not present oscillations in the density values at the stagnation point for the steady state solution. These results justify using $\beta=0.50$

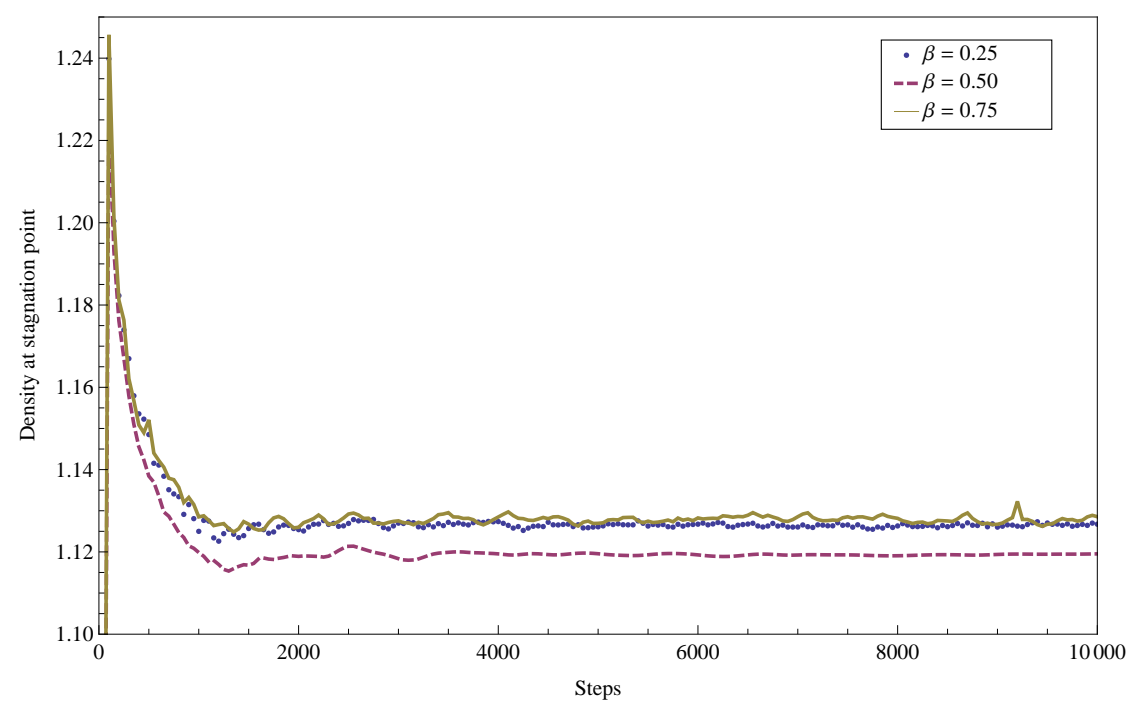

Figure 10: Subsonic viscous flow past a NACA0012 airfoil. Convergence of the density at the stagnation point for different values of $\beta$.

\subsection{Example III: Supersonic viscous flow over flat plate}

The Carter's flat plate example with the flow conditions of $R e=1000, M_{\infty}=3.0$ and $\alpha=0^{\circ}$ is selected here to examine the capability of the current method in the presence of shock waves and boundary layers. The rectangular domain considered with the dimensions of 1.4 and 0.8 along the $x$ and $y$ directions, respectively, is presented in Figure 11. The leading edge of the plate is located at $x=0.2$ and $y=0.0$. The Reynolds number is calculated based on the free stream values and the length in the $x$ direction. A structured mesh is created by dividing the domain in 64 and 112 parts in the $x$ and the $y$ directions, respectively. 


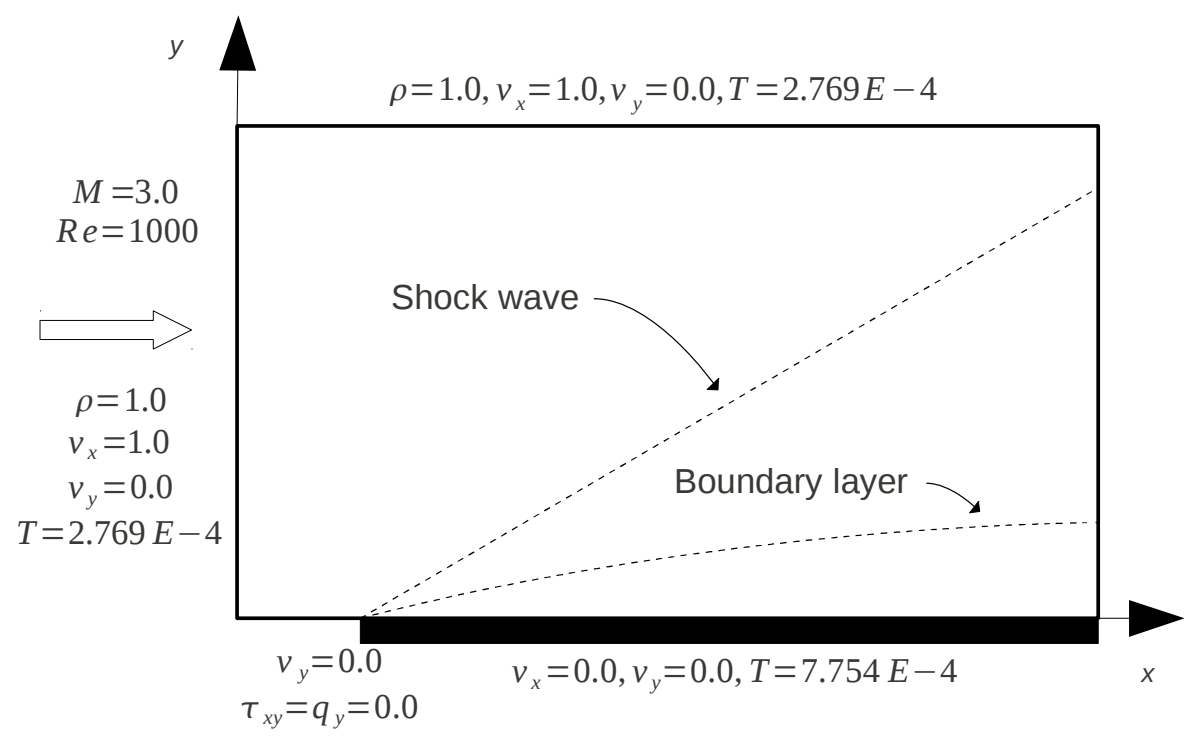

Figure 11: Supersonic flow over flat plate. Problem definition.

As shown in Figure 11, all the values of $\rho, v_{x}, v_{y}$ and $T$ are fixed at the inflow and upper sides of the domain since these boundaries behave as a supersonic inlet. The no-slip boundary condition is applied on the surface of the plate, whereas the stagnation temperature of

$$
T_{\text {stag }}=T_{\infty}\left(1+\frac{\gamma-1}{2} M_{\infty}^{2}\right)
$$

is imposed there, as well. Although a prescription of the density is needed at the subsonic part of the outflow boundary, the flow variables are left free there.

The obtained contours of density, pressure, temperature and Mach number are plotted in Figure 12. The results demonstrate the good behavior of the presented formulation in capturing the shock wave and the boundary layer. 


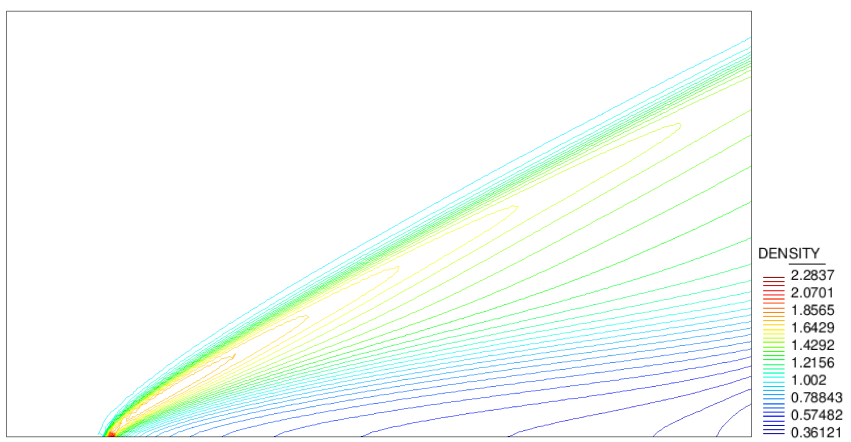

(a)

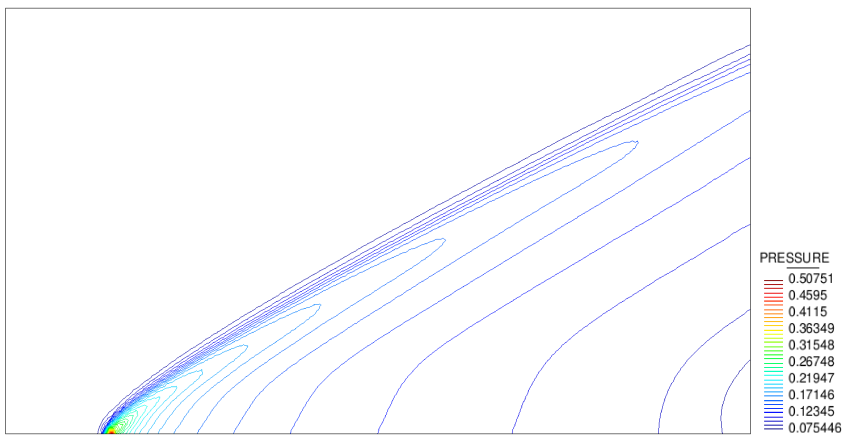

(b)

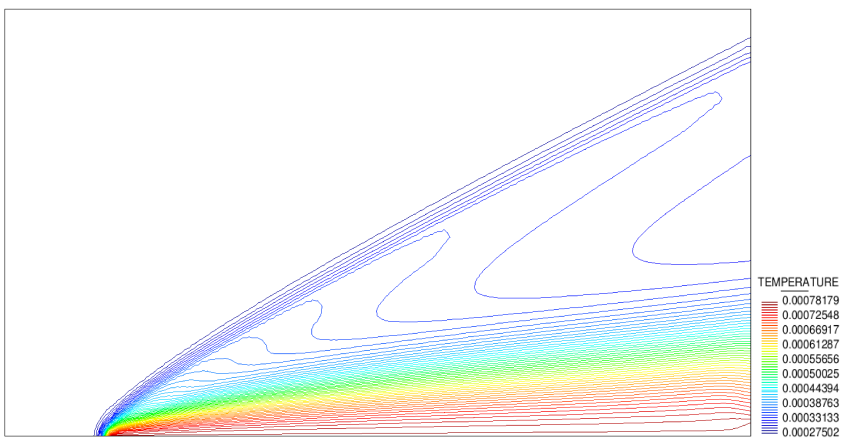

(c)

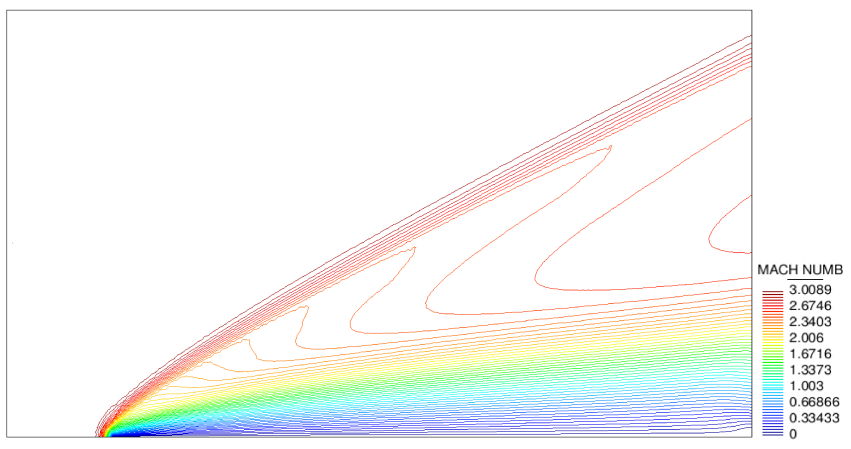

(d)

Figure 12: Supersonic flow over flat plate. (a) density, (b) pressure, (c) temperature and (d) Mach number contours.

The obtained density value and the $y$ component of the velocity along the line $x=1.2$ are compared in Figures 13a and 13b, respectively, with the results presented in [5]. Although the 
obtained peak point values of both the density and $y$ component of the velocity are not coincident with the reference ones, an overall good agreement with the reference results can be observed.

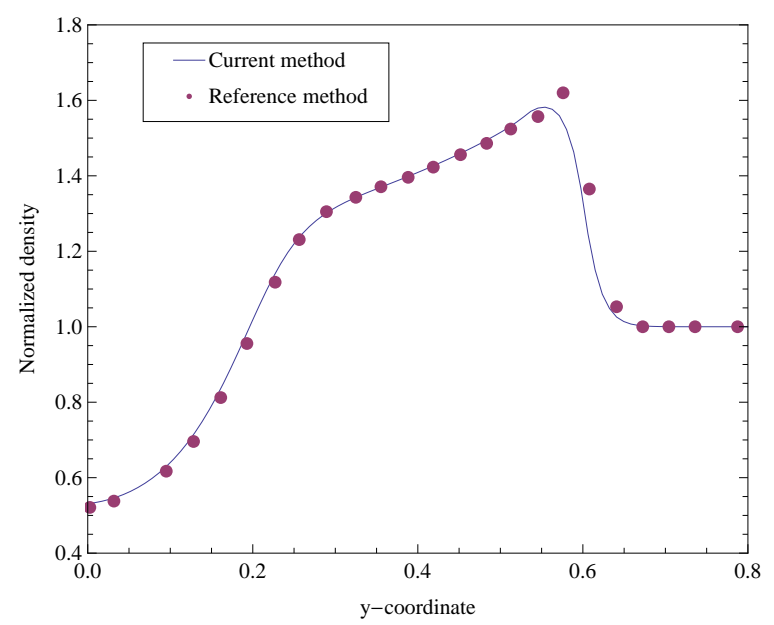

(a)

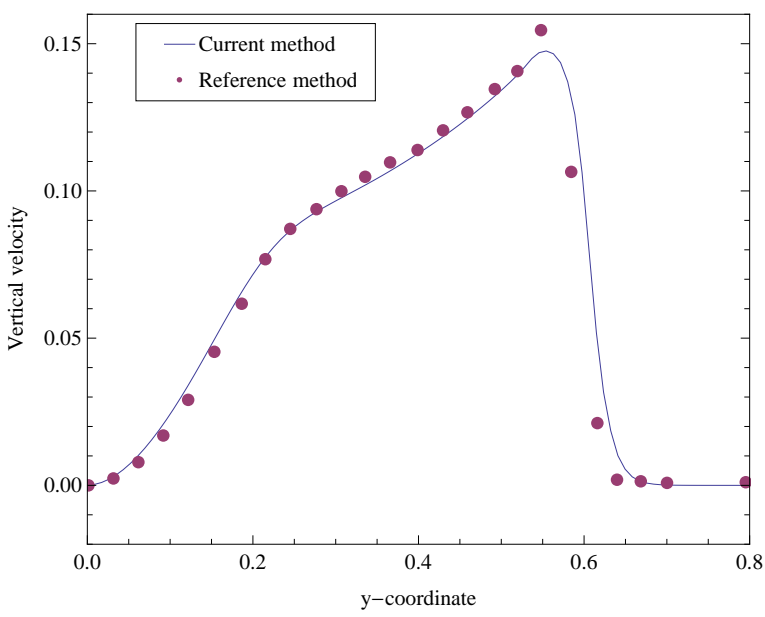

(b)

Figure 13: Supersonic flow over flat plate. Comparison of the obtained (a) density and (b) vertical velocity values along the line $x=1.2$ with the reference results [5].

\subsection{Example IV: Compression corner}

This example is another benchmark of the FIC-FEM formulation for supersonic viscous regimes. The problem data is extracted from [5] where the flow for $R e=16800, M_{\infty}=3.0$ and $\alpha=0^{\circ}$ enters the domain passing over a flat plate and then over a compression corner of $10^{\circ}$ inducing the shock wave and the boundary layer initiated from the leading edge of the plate. The Reynolds number is calculated using the free stream values and the distance between the leading edge of the plate and the compression corner.

Figure 14 schematically shows the computational domain of $0.0 \leq x \leq 1.9$ and $0.0 \leq y \leq 0.716$. The leading edge of the flat plate is located at $x=0.1$ and the compression corner starts from $x=1.1$.

The density, velocity and temperature values are fixed at the inflow and upper boundaries where no condition is prescribed on the outflow boundary. On the plate surface, the no-slip boundary condition, as well as the specification of the temperature as the stagnation temperature, calculated from Equation 39, are applied. 


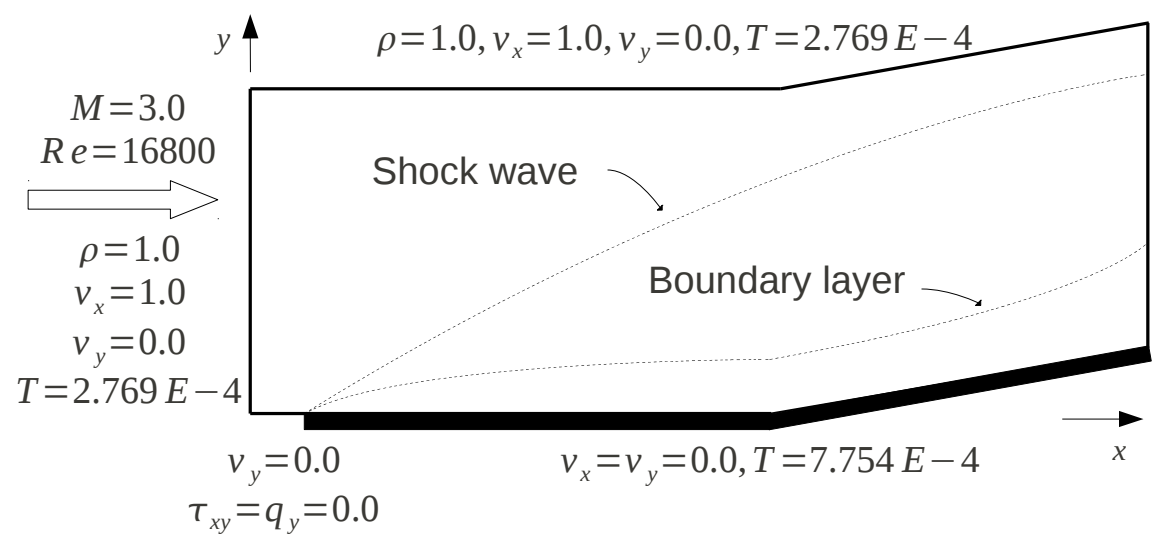

Figure 14: Compression corner. Problem definition.

The domain is discretized using a structured mesh of 3-noded triangles containing 200 points in the streamline direction, and 50 points in the vertical direction where the minimum element size above the plate is taken as 0.0011 giving the maximum aspect ratio of almost 10 (Figure 15).

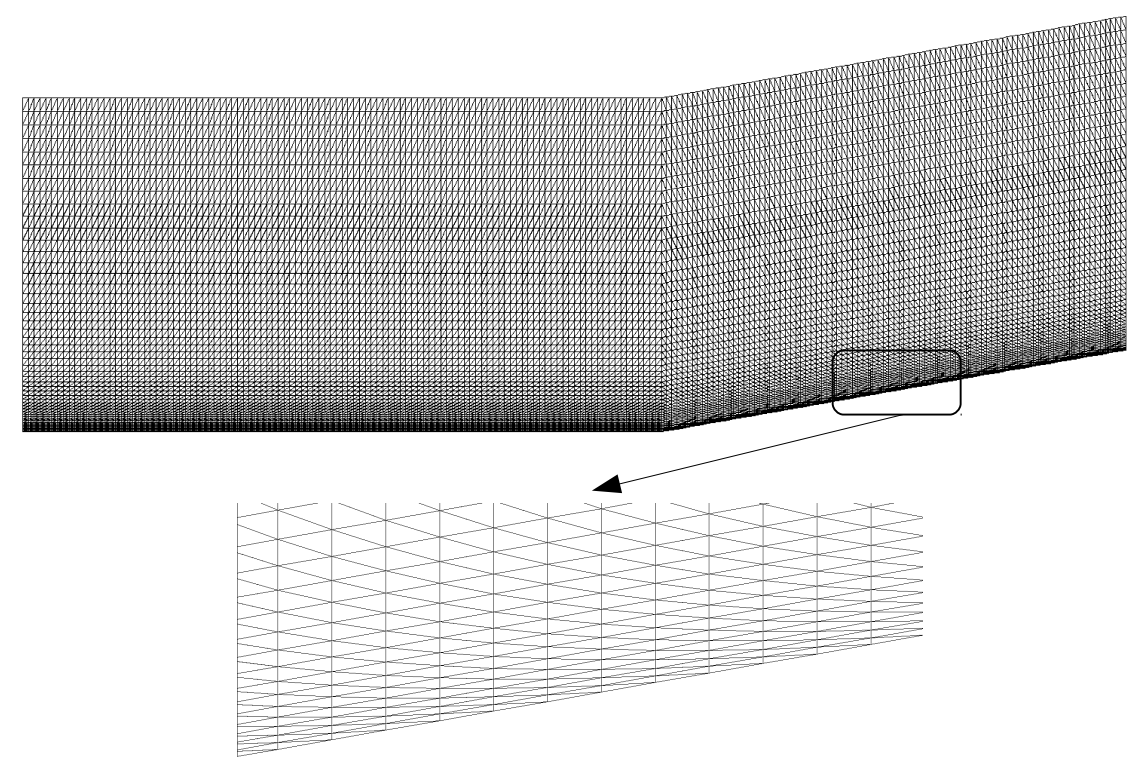

Figure 15: Compression corner. Detail of the structured mesh.

The obtained values for the density, pressure, temperature and Mach number contours are presented in Figure 16. The figure shows that the FIC-FEM approach presented in this work is able to provide smooth results in all the domain, especially near the shock wave and near the boundary layer. The only inaccuracy observed in the results is the presence of non-realistic values at the zone close to the stagnation point which is a point of singularity. It can be seen that the weakness of the formulation in determining the temperature at the stagnation point results in an overestimation of the Mach number there. This problem can be resolved by using elements with less aspect ratio around that region.

The obtained $C_{p}$ and $C_{f}$ distributions along the plate surface are compared to the ones presented by Carter [5] in Figure 17 and Figure 18, respectively. Generally, a good agreement is observed except for the peak values at the stagnation point, as mentioned before. The location of the separation point happens at $x=0.89$ showing an appropriate compatibility with the results presented in the $[5,21,45]$ ranging from $x=0.84$ to $x=0.89$. 


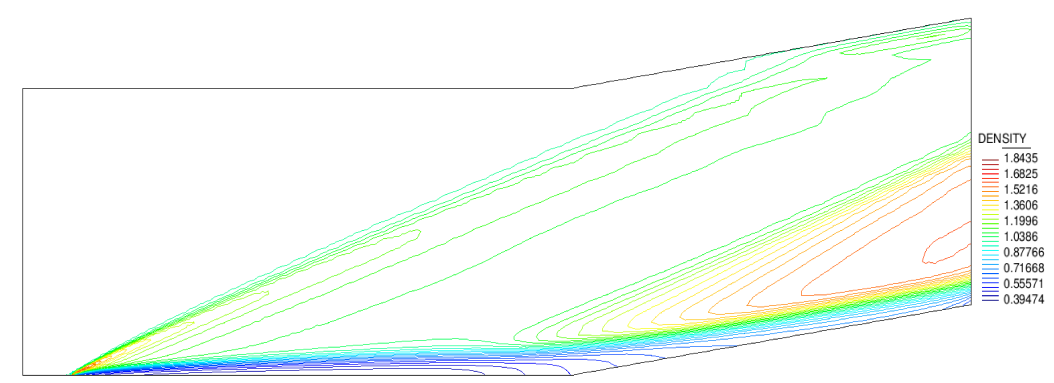

(a)

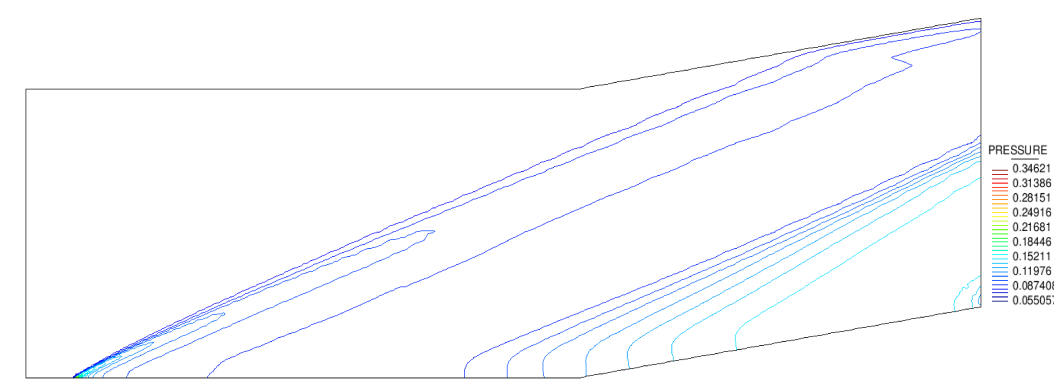

(b)

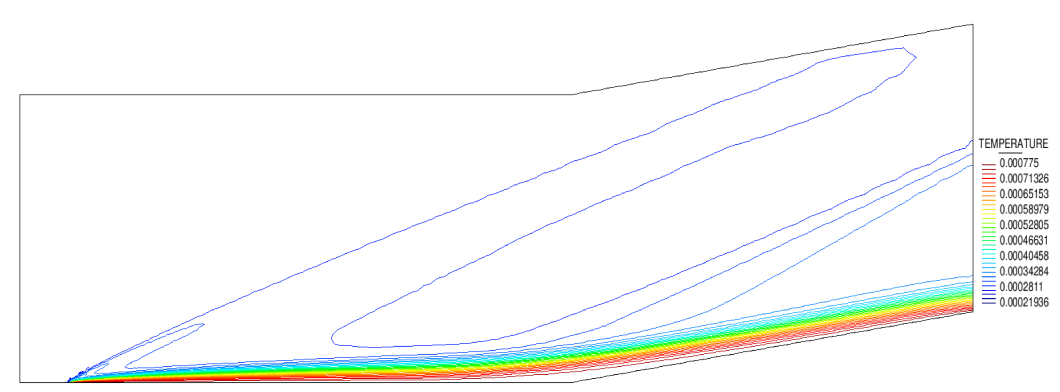

(c)

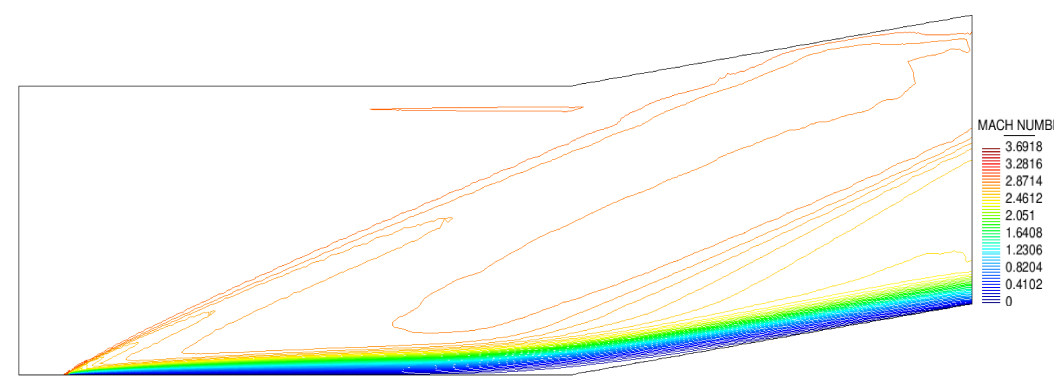

(d)

Figure 16: Compression corner. (a) density, (b) pressure, (c) temperature and (d) Mach number contours. 


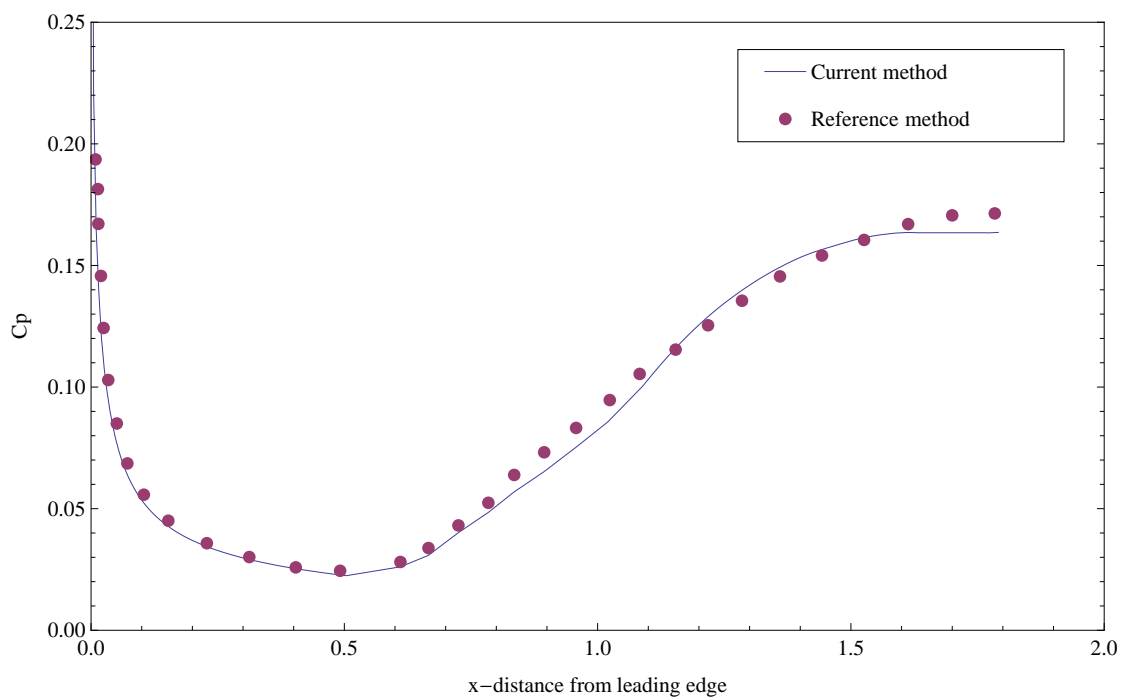

Figure 17: Compression corner. Comparison of the obtained pressure coefficient $C_{p}$ distribution with the numerical results of reference [5].

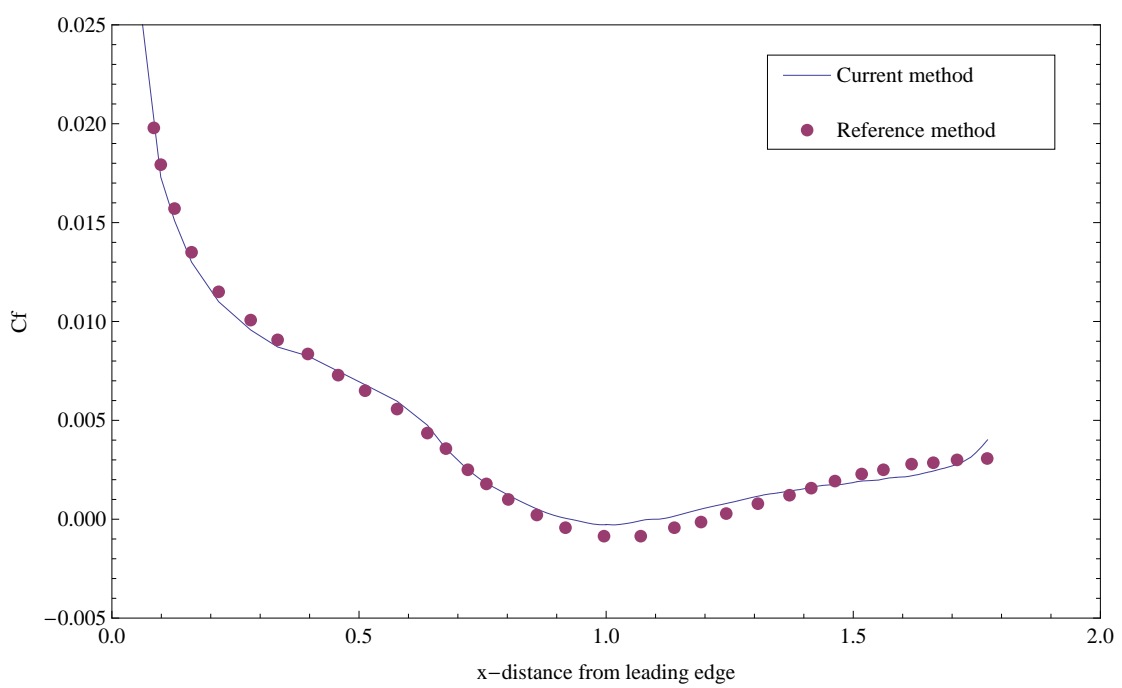

Figure 18: Compression corner. Comparison of the obtained skin-friction coefficient $C_{f}$ distribution with the numerical results of reference [5].

\section{Concluding Remarks}

An implicit stabilized formulation based on the FIC method has been developed for solving the laminar compressible Navier-Stokes equations on unstructured grids using the Galerkin FEM. The arisen non-linear system of equations for the steady state problems is solved by implementing a damped Newton method in conjunction with a preconditioned GMRES method for solving the resulted linear system of equation at each iteration. The capability of the developed FICFEM stabilized formulation has been assessed by introducing several inviscid and viscous test examples. Having compared the numerical results with reference ones, it is found that stable and accurate solutions have been obtained. In particular, the boundary layer is captured as well as the appropriate pressure coefficient $C_{p}$ distribution and the skin-friction coefficient $C_{f}$ distribution along the boundary. In future work, the accuracy of the formulation for estimating the temperature inside the elements with high aspect ratio around the stagnation point needs 
to be enhanced. We also plan to develop the proposed method for 3D applications considering unsteady flows and turbulence effects.

\section{Acknowledgments}

The first author would like to acknowledge the financial support provided by CIMNE. We express our gratitude to Drs. Roberto Flores and Enrique Ortega for helpful discussions and suggestions.

This research was partially supported by the SAFECON project of the European Research Council.

\section{References}

[1] S.K. Aliabadi, S.E. Ray and T.E. Tezduyar, SUPG finite element computation of viscous compressible flows based on the conservation and entropy variables formulations, Computational Mechanics, 11:300-312, 1993.

[2] W.K. Anderson, J.L. Thomas, and B. Van Leer. Comparison of finite volume flux vector splittings for the Euler equations. AIAA Journal, 24 (9):1453-1460, 1986.

[3] J. P. Boris and D. L. Book. Flux corrected transport I. SHASTA, a fluid transport algorithm that works. J. Comput. Phys., 11:38-69, 1973.

[4] A.N. Brooks and T.J.R. Hughes. Streamline upwind/Petrov-Galerkin formulations for convection dominated flows with particular emphasis on the incompressible Navier-Stokes equations. Comput. Methods Appl. Mech. Engrg, 32:199-259, 1982.

[5] J.E. Carter. Numerical solutions of the Navier-Stokes equations for the supersonic laminar flow over a two-dimensional compression corner. NASA Technical Report, TR R-385, 1972.

[6] L. Catabriga, A.L.G.A. Coutinho and T.E. Tezduyar, Compressible Flow SUPG stabilization parameters computed from element-edge matrices, Computational Fluid Dynamics Journal, 13:450-459, 2004.

[7] L. Catabriga, A.L.G.A. Coutinho and T.E. Tezduyar, Compressible flow SUPG parameters computed from degree-of-freedom submatrices, Computational Mechanics, 38:334-343, 2006.

[8] F. Chalot and T.J.R. Hughes. A consistent equilibrium chemistry algorithm for hypersonic flows. Comput. Methods Appl. Mech. Engrg, 112:25-40, 1994.

[9] A. J. Chorin. Numerical solution of the Navier-Stokes equations. Math. Comput., 22:745-762, 1968.

[10] A. J. Chorin. Sur l'approximation de la solution des equations de Navier-Stokes par la methode des pas fractionaires (I). Arch. Rat. Mech. Anal., 32:135-153, 1969.

[11] R. Codina, M. Vazquez, and O.C. Zienkiewicz. A general algorithm for compressible and incompressible flow. Part III: The semi-implicit form. Int. J. Numer. Methods Fluids., 27:1332, 1998.

[12] R. Codina. A discontinuity-capturing crosswind-dissipation for the finite element solution of the convection diffusion equation. Comput. Methods Appl. Mech. Eng, 110:325-342, 1993.

[13] J. Donea. A Taylor-Galerkin method for convective transport problems. Int. J. Numer. Methods Engrg, 20:101-120, 1984.

[14] J. Donea and A. Huerta. Finite Element Methods for Flow Problems. Wiley, 2003.

[15] G. Hauke and T.J.R. Hughes. A unified approach to compressible and incompressible flows. Comput. Methods Appl. Mech. Engrg, 113:389-395, 1994. 
[16] C. Hirsch. Numerical Computation of Internal and External Flow, Vol. 1. J. Wiley, New York, 1988 (vol. 2, 1990).

[17] T.J.R. Hughes and T.E. Tezduyar. Finite element methods for first-order hyperbolic systems with particular emphasis on the compressible Euler equations. Comput. Methods Appl. Mech. Engrg, 45:217-284, 1984.

[18] T.J.R. Hughes, L.P. Franca, and M. Mallet. A new finite element formulation for computational fluid dynamics: VI. Convergence analysis of the generalized SUPG formulation for linear time-dependent multi-dimensional advective-diffusive systems. Comput. Methods Appl. Mech. Engrg, 63:97-112, 1987.

[19] T.J.R. Hughes. Greens functions, the Dirichlet-to-Neumann formulation, subgrid scale models, bubbles and the origins of stabilized method. Comp. Meth. Appl. Mech. Eng, 127:387-401, 2007.

[20] T.J.R. Hughes, G. Scovazzi and T.E. Tezduyar, Stabilized methods for compressible flows", Journal of Scientific Computing, 43:343-368, 2010.

[21] C.M. Hung and R.W. MacCormack. Numerical solutions of supersonic and hypersonic laminar compression corner flows. AIAA J., 14 (4):475-481, 1976.

[22] A. Jameson, W. Schmidt, and E. Turkel. Numerical solution of the Euler equations by finite volume methods using Runge-Kutta time stepping schemes. AIAA paper, 81-1259, June 1981.

[23] B. S. Kirk and G. F. Carey. Development and Validation of a SUPG Finite Element Scheme for the Compressible Navier-Stokes Equations using a Modified Inviscid Flux Discretization. Int. J. Num. Meth. Fluids, 57(3):265-293, 2008.

[24] M. Kouhi and E. Oñate. A stabilized finite element formulation for high-speed inviscid compressible flows using Finite Calculus. Int. J. Num. Meth. Fluids, 74(2):872-897, 2014.

[25] G.J. Le Beau, S.E. Ray, S.K. Aliabadi and T.E. Tezduyar, SUPG finite element computation of compressible flows with the entropy and conservation variables formulations, Comput. Meth. Appl. Mech. Engng., 104:397-422, 1993.

[26] R. Löhner, K. Morgan, J. Peraire, and M. Vahdati. Finite element flux-corrected transport (FEM-FCT) for the Euler and Navier-Stokes equations. Int. J. Num. Methods Fluids, 7:103109, 1987.

[27] D.J. Mavriplis, A. Jameson, and L. Martinelli. Multigrid solution of the Navier-Stokes equations on triangular meshes. ICASE Rep. No. 89-11, 1989.

[28] S. Mittal and T. Tezduyar, A unified finite element formulation for compressible and incompressible flows using augmented conservation variables, Comput. Meth. Appl. Mech. Engng., 161:229-243, 1998.

[29] K. Morgan, J. Peraire, J. Peiro, and O.C. Zienkiewicz. Adaptive remeshing applied to the solution of a shock interaction problem on a cylindrical leading edge. in P. Stow (ed.), Computational Methods in Aeronautical Fluid Dynamics, Clarenden Press, Oxford, pages 327-344, 1990 .

[30] E. Oñate. Derivation of stabilized equations for advective-diffusive transport and fluid flow problems. Comput. Meth. Appl. Mech. Engng., 151:233-267, 1998.

[31] E. Oñate, J. Garcia, and S. Idelsohn. An alpha-adaptive approach for stabilized finite element solution of advective-diffusive problems with sharp gradients. New Adv. in Adaptive Comp. Met. in Mech., P. Ladeveze and J.T. Oden (Eds.), Elsevier, 1998.

[32] E. Oñate and M. Manzan. A general procedure for deriving stabilized space-time finite element methods for advective-diffusive problems. Int. J. Num. Meth. Fluids., 31:203-221, 1999. 
[33] E. Oñate. A stabilized finite element method for incompressible viscous flows using a finite increment calculus formulation. Comp. Meth. Appl. Mech. Eng, 182:355-370, 2000.

[34] E. Oñate and J. Garcia. A finite element method for fluid-structure interaction with surface waves using a finite calculus formulation. Comput. Meth. Appl. Mech. Eng., 191:635-660, 2001.

[35] E. Oñate. Possibilities of finite calculus in computational mechanics. Int. J. Num. Meth. Engng, 60:255-281, 2004.

[36] E. Oñate, A. Valls, J. Garcia. Computation of turbulent flows using a finite calculus-finite element formulation. Int. J. Numer. Meth. Engng., 54:609-637, 2007.

[37] E. Oñate, A. Valls, J. Garcia. Modeling incompressible flows at low and high Reynolds numbers via a finite calculus-finite element approach. Journal of Computational Physics, 224:332$351,2007$.

[38] J. Peraire, J. Peiro, L. Formaggia, K. Morgan, and O.C. Zienkiewicz. Finite element Euler computations in three dimensions. Int. J. Numer. Methods Eng., 26:2135-2159, 1988.

[39] Y. Saad and M. H. Schultz. GMRES: A Generalized Minimum Residual Algorithm for Solving Nonsymmetric Linear Systems. SIAM Journal of Scientific and Statistical Computing, 7:856$869,1986$.

[40] G. Scovazzi. A discourse on Galilean invariance, SUPG stabilization, and the variational multiscale framework. Comp. Meth. Appl. Mech. Eng, 196(4-6):1108-1132, 2007.

[41] G. Scovazzi. Galilean invariance and stabilized methods for compressible flows. Int. J. Num. Meth. Fluids, 54(6-8):757-778, 2007.

[42] G. Scovazzi, M.A. Christon, T.J.R. Hughes, and J.N. Shadid. Stabilized shock hydrodynamics: I. A Lagrangian method. Comp. Meth. Appl. Mech. Eng, 196(4-6):923-966, 2007.

[43] G. Scovazzi, J.N. Shadid, E. Love, and W.J. Rider. A conservative nodal variational multiscale method for Lagrangian shock hydrodynamics. Comp. Meth. Appl. Mech. Eng, 199(4952):3059-3100, 2010.

[44] G. Scovazzi. Lagrangian shock hydrodynamics on tetrahedral meshes. A stable and accurate variational multiscale approach. J. Comput. Phys., 231:8029-8069, 2012.

[45] F. Shakib. Finite element analysis of the compressible Euler and Navier-Stokes equations. PhD thesis, Department of Mechanical Engineering, Stanford University, 1988.

[46] F. Shakib, T.J.R. Hughes, and Z. Johan. A new finite element formulation for computational fluid dynamics: X. The compressible Euler and Navier-Stokes equations. Comput. Methods Appl. Mech. Engrg, 89:141-219, 1991.

[47] T.E. Tezduyar and M. Senga, Stabilization and shock-capturing parameters in SUPG formulation of compressible flows, Comput. Methods Appl. Mech. Engrg, 195:1621-1632, 2006.

[48] E. Turkel. Improving the accuracy of central difference schemes. ICASE Report 8853, 1988.

[49] O.C. Zienkiewicz and J. Wu. A general explicit of semi-explicit algorithm for compressible and incompressible flows. Int. J. Numer. Methods Eng., 35:457-479, 1992.

[50] O.C. Zienkiewicz and R. Codina. A general algorithm for compressible and incompressible flow. Part I. The split characteristic based scheme. Int. J. Numer. Methods Fluids., 20:869$885,1995$.

[51] O.C. Zienkiewicz, B.V.K. Satya Sai, K. Morgan, R. Codina, and M. Vazquez. A general algorithm for compressible and incompressible flow. Part II. Tests on the explicit form. Int. J. Numer. Methods Fluids., 20:887-913, 1995.

[52] O.C. Zienkiewicz, R.L. Taylor, and P. Nithiarasu. The Finite Element Method. Vol. 3 Fluid Dynamics. Elsevier, 6th Edition, 2005. 

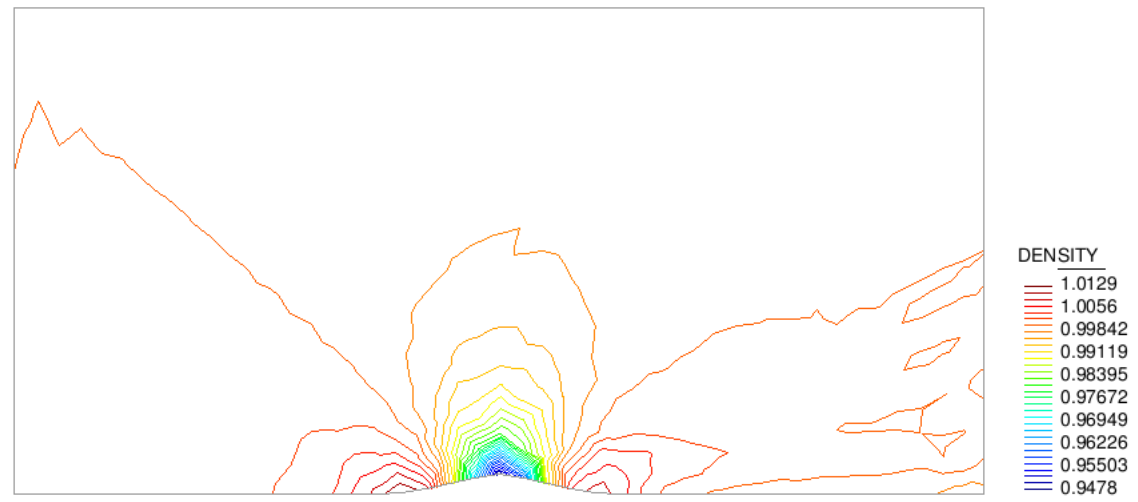

(a)
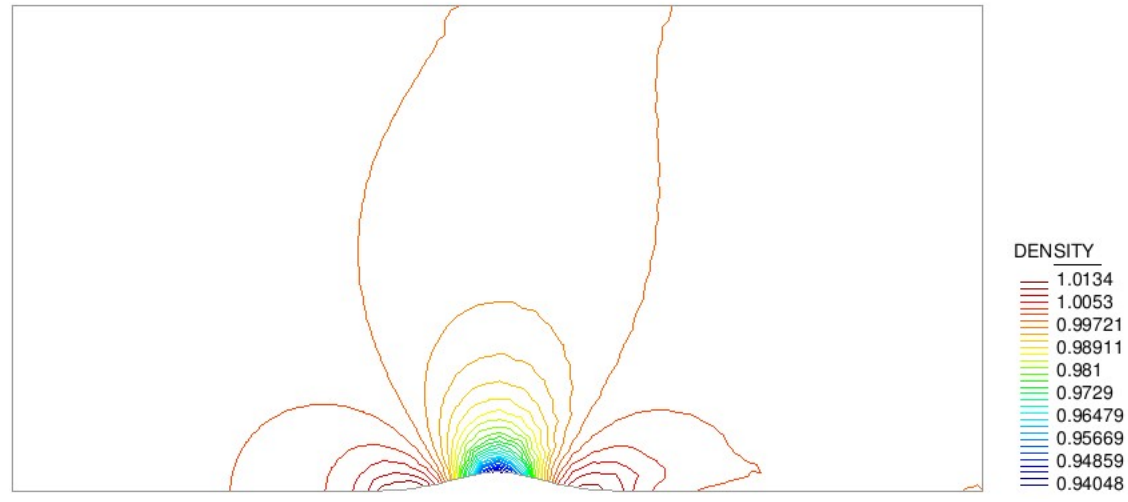

(b)
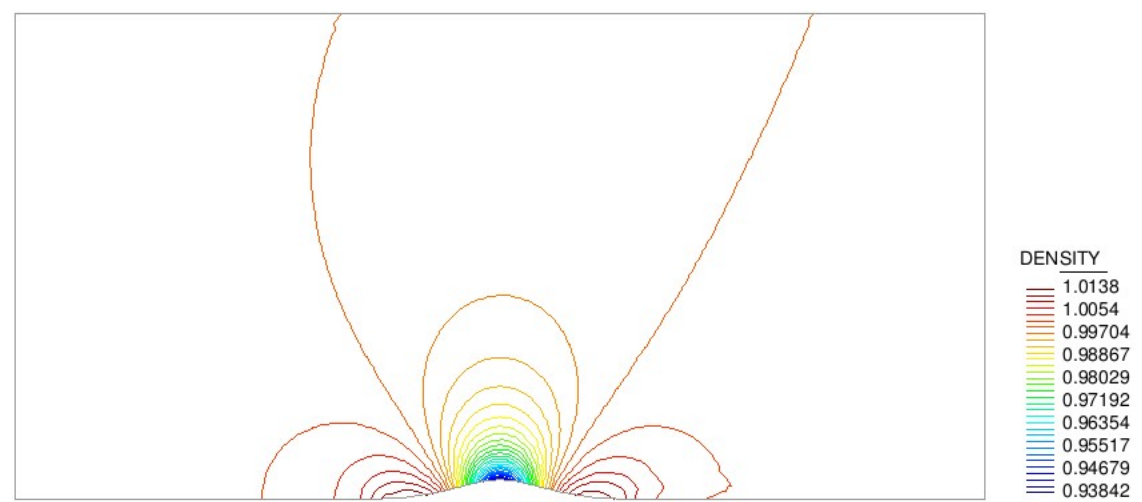

(c)

Figure 2: Subsonic inviscid flow past a bump example. Density contours for (a) coarse mesh, (b) intermediate mesh and (c) fine mesh. 